\title{
Thermodynamics of the polaron master equation at finite bias
}

\author{
Thilo Krause, ${ }^{1, a)}$ Tobias Brandes, ${ }^{1}$ Massimiliano Esposito, ${ }^{2}$ and Gernot Schaller ${ }^{1, b)}$ \\ ${ }^{1}$ Institut für Theoretische Physik, Technische Universität Berlin, Hardenbergstr. 36, D-10623 Berlin, Germany \\ ${ }^{2}$ Complex Systems and Statistical Mechanics, University of Luxembourg, L-1511 Luxembourg, Luxembourg
}

(Received 23 September 2014; accepted 17 March 2015; published online 3 April 2015)

\begin{abstract}
We study coherent transport through a double quantum dot. Its two electronic leads induce electronic matter and energy transport and a phonon reservoir contributes further energy exchanges. By treating the system-lead couplings perturbatively, whereas the coupling to vibrations is treated non-perturbatively in a polaron-transformed frame, we derive a thermodynamic consistent lowdimensional master equation. When the number of phonon modes is finite, a Markovian description is only possible when these couple symmetrically to both quantum dots. For a continuum of phonon modes however, also asymmetric couplings can be described with a Markovian master equation. We compute the electronic current and dephasing rate. The electronic current enables transport spectroscopy of the phonon frequency and displays signatures of Franck-Condon blockade. For infinite external bias but finite tunneling bandwidths, we find oscillations in the current as a function of the internal bias due to the electron-phonon coupling. Furthermore, we derive the full fluctuation theorem and show its identity to the entropy production in the system. (C) 2015 AIP Publishing LLC. [http://dx.doi.org/10.1063/1.4916359]
\end{abstract}

\section{INTRODUCTION}

Electronic transport through low-dimensional systems, e.g., quantum dots or molecular junctions, has been a vivid research field over the last years. In part, this has been triggered by the fact that single molecules or quantum dot configurations are promising candidates for a variety of applications such as, e.g., charge ${ }^{1}$ and $\operatorname{spin}^{2}$ qubits or single photon emitters which are, for example, realized in semiconductor nanowires. ${ }^{3,4}$ For efficient device performance, a detailed understanding of electronic interplay with its environment, e.g., optical modes, ${ }^{5}$ is important. In particular, the interaction with vibrational modes has been studied in order to reveal quantum phenomena such as additional decoherence. ${ }^{6-9}$ Moreover, phonon spectroscopy ${ }^{10-13}$ can be used to visualize quantum effects in transport characteristics such as Franck-Condon blockade and giant Fano factors. ${ }^{14-21}$ It was also proposed to use biascontrolled electronic transport to selectively excite vibrational modes. ${ }^{22}$ Furthermore, also from a more classical perspective, the study of thermo-electric effects in phonon-coupled nanojunctions ${ }^{23-27}-$ e.g., the conversion of heat to work ${ }^{28-31}$ or local cooling ${ }^{32-34}$-leads to interesting new questions.

A crucial parameter for understanding many of these effects is the coupling strength between electronic transport and phonon modes. Therefore, weak ${ }^{35,36}$ and strong ${ }^{37-41}$ coupling regimes have been studied with different methods. Recently, the possibility to tune the coupling strength in the experiment has been suggested. ${ }^{42}$

Even though most authors consider simplified models consisting of a few-level-system coupled to either a single (Anderson-Holstein model) ${ }^{43-46}$ or multiple ${ }^{47}$ phonon modes,

\footnotetext{
a)Electronic address: tkrause@physik.tu-berlin.de

b)Electronic address: gernot.schaller@tu-berlin.de
}

the inclusion of the vibration dynamics in the system leads to an infinite-dimensional Hilbert space and therefore large computational complexity. This problem has triggered the development of many different methods, such as master equations, ${ }^{27,48}$ full counting statistics approaches, ${ }^{49-51}$ Lindblad kinetic equations, ${ }^{52}$ Greens functions, ${ }^{37,53}$ or higher-order methods. ${ }^{54}$ For time-dependent studies, often the multilayer multiconfiguration time-dependent Hartree method ${ }^{55-57}$ or real-time path integral approaches are applied. ${ }^{58-60}$ Additional complexity arises if intrinsic tunneling between multiple system states and their coupling to phonon modes is taken into account. Among the conceptually simple master equation approaches, the polaron-transformed master equation ${ }^{61}$ often allows a simple diagonalization of the system Hamiltonian, which has renewed interest in the phonon master equation in the finite-bias regime. ${ }^{62,63}$ As long as the phonons are contained within the system, this leads to thermodynamic consistency but does not solve the curse of dimensionality.

When, in contrast, within a strong electron-phononcoupling scenario the vibrations are treated as part of a reservoir, thermodynamic consistency is non-trivial even for a single electronic level. ${ }^{64}$ Here, the proof of the fluctuation theorem ${ }^{65}$ offers a well known tool because it directly confirms the second law of thermodynamics. ${ }^{66-68}$ In particular, in this paper we discuss the derivation of a phonon master equation for a double quantum dot model, introduced in Sec. II, coupled to macroscopic electronic leads and either a discrete or continuous phonon reservoir. Even in absence of phonons, we explicitly allow for electronic tunneling within the system. We treat the phonons as part of a non-standard reservoir yielding a finite system Hilbert space and, thus, a low dimensional master equation with minimized computational requirements making the method applicable for the study of even larger systems. We put emphasis on the polaron transformation and its effect on 
the model Hamiltonian in terms of thermodynamic consistency. Staying in the polaron picture, we present a detailed derivation of the quantum master equation, see Sec. III, and prove its thermodynamic consistency by deriving the fluctuation theorem in Sec. IV. Finally, in Sec. V, we analyze electronic current and dephasing rate for particular physical situations showing a Franck-Condon-like suppression in both quantities. We also investigate the possibility of phonon spectroscopy experiments. In addition, we discuss the performance of the model system as a thermoelectric generator converting a temperature gradient into useful power.

\section{MODEL}

\section{A. Hamiltonian}

We consider a system made of a double quantum dot (DQD) in contact with multiple reservoirs $\mathcal{H}=\mathcal{H}_{\mathrm{S}}+\mathcal{H}_{\mathrm{B}}$ $+\mathcal{H}_{\mathrm{SB}}$. The reservoirs $\mathcal{H}_{\mathrm{B}}=\mathcal{H}_{\mathrm{B}}^{\mathrm{el}}+\mathcal{H}_{\mathrm{B}}^{\mathrm{ph}}$ and the system-bath coupling $\mathcal{H}_{\mathrm{SB}}=\mathcal{H}_{\mathrm{SB}}^{\mathrm{el}}+\mathcal{H}_{\mathrm{SB}}^{\mathrm{ph}}$ contain electronic and phonon contributions, respectively. The DQD Hamiltonian reads

$$
\begin{aligned}
\mathcal{H}_{\mathrm{S}} \equiv & \varepsilon_{\mathrm{L}} d_{\mathrm{L}}^{\dagger} d_{\mathrm{L}}+\varepsilon_{\mathrm{R}} d_{\mathrm{R}}^{\dagger} d_{\mathrm{R}}+T_{\mathrm{c}}\left(d_{\mathrm{L}} d_{\mathrm{R}}^{\dagger}+d_{\mathrm{R}} d_{\mathrm{L}}^{\dagger}\right) \\
& +U d_{\mathrm{L}}^{\dagger} d_{\mathrm{L}} d_{\mathrm{R}}^{\dagger} d_{\mathrm{R}},
\end{aligned}
$$

where $d_{\sigma}\left(d_{\sigma}^{\dagger}\right)$ annihilates (creates) an electron in dot $\sigma$ with on-site energy $\varepsilon_{\sigma}(\sigma \in\{\mathrm{L}, \mathrm{R}\}$ throughout this paper $), T_{\mathrm{c}}$ is the internal electronic tunneling amplitude, and $U$ is the Coulomb repulsion energy. The system is connected to two electronic leads, left and right, held at thermal equilibrium

$$
\mathcal{H}_{\mathrm{B}}^{\mathrm{el}} \equiv \sum_{k} \sum_{\sigma \in\{\mathrm{L}, \mathrm{R}\}} \varepsilon_{k, \sigma} c_{k, \sigma}^{\dagger} c_{k, \sigma}
$$

Here, the fermionic operator $c_{k, \sigma}\left(c_{k, \sigma}^{\dagger}\right)$ annihilates (creates) electrons in mode $k$ with energy $\varepsilon_{k, \sigma}$. Note that we do not distinguish between the electronic spins, which implicitly assumes that, e.g., the leads are completely polarized. Electronic transport through the system is enabled by the dot-lead interaction Hamiltonian

$$
\mathcal{H}_{\mathrm{SB}}^{\mathrm{el}} \equiv \sum_{k, \sigma \in\{\mathrm{L}, \mathrm{R}\}}\left(t_{k, \sigma} d_{\sigma} c_{k, \sigma}^{\dagger}+\text { h.c. }\right)
$$

with the tunneling amplitudes $t_{k, \sigma}$ (which we will treat perturbatively to second order later-on).

Additionally, the system is coupled to a bosonic reservoir

$$
\mathcal{H}_{\mathrm{B}}^{\mathrm{ph}} \equiv \sum_{q} \omega_{q} a_{q}^{\dagger} a_{q}
$$

with phonon operator $a_{q}\left(a_{q}^{\dagger}\right)$ annihilating (creating) a phonon in mode $q$ with frequency $\omega_{q}$. The electronic occupation of the system induces vibrations in the phonon bath via the electronphonon interaction Hamiltonian

$$
\mathcal{H}_{\mathrm{SB}}^{\mathrm{ph}} \equiv \sum_{q} \sum_{\sigma \in\{\mathrm{L}, \mathrm{R}\}}\left(h_{q, \sigma} a_{q}+\text { h.c. }\right) d_{\sigma}^{\dagger} d_{\sigma},
$$

with the phononic absorption/emission amplitudes $h_{q, \sigma}$ (which we want to treat non-perturbatively later-on).

\section{B. Polaron transformation}

In order to investigate the impact of strong electronphonon coupling on electronic transport we perform the unitary Lang-Firsov (polaron) transformation, ${ }^{69,70} \overline{\mathcal{H}}=U \mathcal{H} U^{\dagger}$, with the unitary operator $U=e^{d_{\mathrm{L}} d_{\mathrm{L}}^{\dagger} \mathcal{B}_{\mathrm{L}}+d_{\mathrm{R}} d_{\mathrm{R}}^{\dagger} \mathcal{B}_{\mathrm{R}}}$. The antiHermitian operator $\mathcal{B}_{\sigma}$ is defined as

$$
\mathcal{B}_{\sigma} \equiv \sum_{q}\left(h_{q, \sigma}^{*} a_{q}^{\dagger}-h_{q, \sigma} a_{q}\right) / \omega_{q} .
$$

The details of the polaron transformation are shown in Appendix A. After the polaron transformation, the Hamiltonian admits a new decomposition into system, interaction, and reservoir contributions. It is important to note, however, that in general such decompositions are not unique: for example, for a system Hamiltonian $H_{\mathrm{S}}$ and an interaction Hamiltonian of the general form $H_{\mathrm{I}}=\sum_{\alpha} A_{\alpha} \otimes B_{\alpha}$ with system and reservoir operators $A_{\alpha}$ and $B_{\alpha}$, respectively, it is straightforward to see that the transformation $H_{\mathrm{S}} \rightarrow H_{\mathrm{S}}+\sum_{\alpha} \kappa_{\alpha} A_{\alpha}$ and $H_{\mathrm{I}} \rightarrow \sum_{\alpha} A_{\alpha} \otimes\left(B_{\alpha}-\kappa_{\alpha} \mathbf{1}\right)$ with numbers $\kappa_{\alpha}$ leaves the total Hamiltonian invariant.

We resolve this ambiguity by demanding that all thermal equilibrium expectation values of linear bath coupling operators should vanish. We have observed that without imposing this requirement, one would arrive at a thermodynamic inconsistent master equations (e.g., predicting non-vanishing currents at global equilibrium). Consequently, we fix the numbers $\kappa_{\alpha}$ as

$$
\kappa_{\alpha}=\left\langle B_{\alpha}\right\rangle,
$$

where the expectation value has to be taken with respect to a thermal equilibrium state of the reservoir corresponding to $B_{\alpha}$.

With this convention, the total Hamiltonian can then be written as $\overline{\mathcal{H}}=\overline{\mathcal{H}}_{\mathrm{S}}+\overline{\mathcal{H}}_{\mathrm{B}}+\overline{\mathcal{H}}_{\mathrm{SB}}$.

Most simple, the reservoir part of the Hamiltonian remains invariant

$$
\overline{\mathcal{H}}_{\mathrm{B}} \equiv \sum_{k} \sum_{\sigma \in\{\mathrm{L}, \mathrm{R}\}} \varepsilon_{k, \sigma} c_{k, \sigma}^{\dagger} c_{k, \sigma}+\sum_{q} \omega_{q} a_{q}^{\dagger} a_{q} .
$$

The system contribution to the Hamiltonian now experiences modified parameters

$$
\begin{aligned}
\overline{\mathcal{H}}_{\mathrm{S}}= & \bar{\varepsilon}_{\mathrm{L}} d_{\mathrm{L}}^{\dagger} d_{\mathrm{L}}+\bar{\varepsilon}_{\mathrm{R}} d_{\mathrm{R}}^{\dagger} d_{\mathrm{R}}+\bar{U} d_{\mathrm{L}}^{\dagger} d_{\mathrm{L}} d_{\mathrm{R}}^{\dagger} d_{\mathrm{R}} \\
& +\left(\bar{T}_{\mathrm{c}} e^{-2 \mathrm{i} \Phi} d_{\mathrm{L}} d_{\mathrm{R}}^{\dagger}+\bar{T}_{\mathrm{c}}^{*} e^{+2 \mathrm{i} \Phi} d_{\mathrm{R}} d_{\mathrm{L}}^{\dagger}\right),
\end{aligned}
$$

with renormalized on-site energy levels ${ }^{71}$

$$
\bar{\varepsilon}_{\sigma} \equiv \varepsilon_{\sigma}-\sum_{q} \frac{\left|h_{q, \sigma}\right|^{2}}{\omega_{q}}
$$

and renormalized Coulomb repulsion ${ }^{72}$

$$
\bar{U} \equiv U-\sum_{q} \frac{h_{q, \mathrm{~L}}^{*} h_{q, \mathrm{R}}+h_{q, \mathrm{~L}} h_{q, \mathrm{R}}^{*}}{\omega_{q}} .
$$

We note that in the strong-coupling limit, attractive Coulomb interactions $(\bar{U}<0)$ are in principle possible. ${ }^{73,74}$ Furthermore, we observe that also the internal tunneling amplitude is renormalized

$$
\bar{T}_{\mathrm{c}} \equiv T_{\mathrm{c}} \kappa,
$$


where the complex-valued $\kappa$ is defined by $\kappa \equiv\left\langle e^{-\mathcal{B}_{\mathrm{L}}} e^{\mathcal{B}_{\mathrm{R}}}\right\rangle$. Assuming that the phonon reservoir in the polaron-transformed frame is in thermal equilibrium, it (see Appendix B) explicitly evaluates to

$$
\kappa=e^{-\sum_{q} \frac{\left|h_{q, \mathrm{~L}}-h_{q, \mathrm{R}}\right|^{2}}{\omega_{q}^{2}}\left[\frac{1}{2}+n\left(\omega_{q}\right)\right]} e^{+\mathrm{i} \Phi},
$$

containing the Bose-distribution $n_{\mathrm{B}}(\omega)=\left[e^{\beta_{\mathrm{ph}} \omega}-1\right]^{-1}$ with the inverse phonon bath temperature $\beta_{\mathrm{ph}}$. Here, the phase $\Phi$ is defined via

$$
\mathrm{i} \Phi \equiv\left[\mathcal{B}_{\mathrm{L}}, \mathcal{B}_{\mathrm{R}}\right] / 2=\sum_{q} \frac{h_{q, \mathrm{R}}^{*} h_{q, \mathrm{~L}}-h_{q, \mathrm{R}} h_{q, \mathrm{~L}}^{*}}{2 \omega_{q}^{2}} .
$$

Finally, the interaction Hamiltonian $\overline{\mathcal{H}}_{\mathrm{SB}} \equiv \overline{\mathcal{H}}_{\mathrm{V}}+\overline{\mathcal{H}}_{\mathrm{T}}$ is made of two parts. The first describes electronic transitions between system and leads

$$
\begin{aligned}
& \overline{\mathcal{H}}_{\mathrm{V}} \equiv \sum_{k}\left(t_{k, \mathrm{~L}} d_{\mathrm{L}} e^{-d_{\mathrm{R}}^{\dagger} d_{\mathrm{R}}^{\mathrm{i} \Phi}} e^{-\mathcal{B}_{\mathrm{L}}} c_{k, \mathrm{~L}}^{\dagger}+\text { h.c. }\right) \\
& +\sum_{k}\left(t_{k, \mathrm{R}} d_{\mathrm{R}} e^{+d_{\mathrm{L}}^{\dagger} d_{\mathrm{L}}^{\mathrm{i} \Phi}} e^{-\mathcal{B}_{\mathrm{R}}} c_{k, \mathrm{R}}^{\dagger}+\text { h.c. }\right),
\end{aligned}
$$

which are now accompanied by multiple phonon emissions or absorptions. The second part describes transitions between left and right dots

$$
\begin{aligned}
\overline{\mathcal{H}}_{\mathrm{T}} \equiv & T_{\mathrm{c}} e^{-2 \mathrm{i} \Phi} d_{\mathrm{L}} d_{\mathrm{R}}^{\dagger}\left(e^{-\mathcal{B}_{\mathrm{L}}} e^{+\mathcal{B}_{\mathrm{R}}}-\kappa\right) \\
& +T_{\mathrm{c}} e^{+2 \mathrm{i} \Phi} d_{\mathrm{R}} d_{\mathrm{L}}^{\dagger}\left(e^{-\mathcal{B}_{\mathrm{R}}} e^{+\mathcal{B}_{\mathrm{L}}}-\kappa^{*}\right),
\end{aligned}
$$

which are also dressed by multiple phonon excitations, see Eq. (6).

The effect of the polaron transformation is visualized in Fig. 1. The coupling to the phonon modes is no longer linear in the annihilation and creation operators anymore, as can be seen by expanding the exponentials $e^{ \pm \mathcal{B}_{\sigma}}$. Comparing the system Hamiltonians before and after the polaron transformation, we see that apart from the renormalized on-site energies and Coulomb repulsion the electron-phonon interaction also renormalizes the internal tunneling term. Consequently, the energy eigenbasis of $\overline{\mathcal{H}}_{\mathrm{S}}$ is now influenced by the systemreservoir interaction strength in the original frame.

\section{Phonon treatment}

We note that when the internal electronic tunneling amplitude was initially absent $\left(T_{\mathrm{c}}=0\right)$, it would be straightforward to keep the phonons as part of the system: electronphonon interactions would only arise from the electronic jumps to and from the leads, such that diagonalizing the system Hamiltonian would be straightforward. In this case, the resulting thermodynamics would be that of a two-terminal system exchanging matter and energy with the two electronic leads. For finite $T_{\mathrm{c}}$ however, keeping the phonons as part of the system would - to obtain a thermodynamically consistent master equation-require to diagonalize an interacting infinitedimensional Hamiltonian (such that the polaron transformation would be of no use). Therefore, we will proceed differently here and consider the phonons as part of the reservoir. When we enforce the phonons in the polaron frame in a thermal equilibrium state $\propto e^{-\beta_{\mathrm{ph}} \mathcal{H}_{\mathrm{B}}^{\mathrm{ph}}}$, this does in the original frame actually

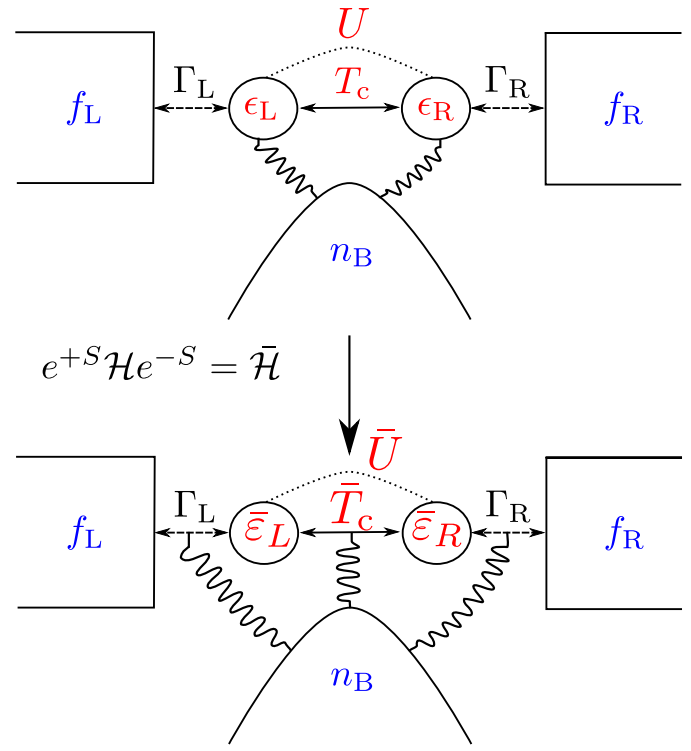

FIG. 1. Sketch of the model before (top) and after (below) the polaron transformation. The double quantum dot system in serial configuration is coupled to electronic leads, left and right, each following Fermi-Dirac statistics with Fermi functions $f_{\mathrm{L}}$ and $f_{\mathrm{R}}$, respectively. If either temperatures or chemical potentials are chosen differently, a non-equilibrium situation is created which enables the exchange of matter and energy between those baths. The tunneling between system and leads is described by the tunneling rates $\Gamma_{L}$ and $\Gamma_{R}$. The quantum tunneling between left and right dot is modulated by the internal tunneling rate $T_{\mathrm{c}}$. Before the polaron transformation (with $S=\sum_{\sigma} d_{\sigma}^{\dagger} d_{\sigma} \mathcal{B}_{\sigma}$ ), the phonon bath with Bose distribution $n_{\mathrm{B}}$ couples directly to the occupation of the quantum dots left and right. Due to the polaron transformation, the coupling is shifted to the electronic jumps which now occur with multiple phonon emission or absorption processes. Another feature of the model in the polaron picture is the renormalization of on-site energies and Coulomb repulsion which now depend on the phonon coupling strength as well as the phonon mode frequency.

correspond to a thermal phonon state that is conditioned on the electronic occupation of the dots, see Appendix C. A similar behaviour would be observed with phonons treated as part of the system, but additionally strongly coupled to another thermal reservoir that imposes fast equilibration of the phonons dependent on the electronic occupation. ${ }^{15,58,60} \mathrm{We}$ are aiming at a thermodynamically consistent description of this extreme limit, where the phonons immediately equilibrate in an electron-dependent thermal state.

\section{MASTER EQUATION IN THE STRONG ELECTRON-PHONON COUPLING LIMIT}

\section{A. Pointer basis}

We do now follow the standard derivation of a master equation, ${ }^{75,76}$ starting from the general decomposition of the interaction Hamiltonian into system $\left(A_{\alpha}\right)$ and bath $\left(B_{\alpha}\right)$ operators (here in the Schrödinger picture),

$$
\mathcal{H}_{\mathrm{SB}}=\sum_{\alpha} A_{\alpha} \otimes B_{\alpha} .
$$

We note that such a tensor product decomposition is possible also for fermionic tunneling terms since one can map the fermionic operators to system and lead fermions via a Jordan-Wigner transform. ${ }^{77}$ Ordering system and bath operators according to Eq. (17), respectively, we obtain 6 coupling 
operators for system

$$
\begin{aligned}
& A_{1}=d_{\mathrm{L}}=\left(A_{2}\right)^{\dagger}, \quad A_{3}=d_{\mathrm{R}}=\left(A_{4}\right)^{\dagger}, \\
& A_{5}=e^{-2 \mathrm{i} \Phi} d_{\mathrm{L}} d_{\mathrm{R}}^{\dagger}=\left(A_{6}\right)^{\dagger},
\end{aligned}
$$

and reservoir

$$
\begin{aligned}
& B_{1}=\sum_{k} t_{k, \mathrm{~L}} c_{k, \mathrm{~L}}^{\dagger} e^{-\mathcal{B}_{\mathrm{L}}}=B_{2}^{\dagger}, \\
& B_{3}=\sum_{k}^{k} t_{k, \mathrm{R}} c_{k, \mathrm{R}}^{\dagger} e^{-\mathcal{B}_{\mathrm{R}}}=B_{4}^{\dagger}, \\
& B_{5}=e^{-\mathcal{B}_{\mathrm{L}}} e^{+\mathcal{B}_{\mathrm{R}}}-\kappa=B_{6}^{\dagger} .
\end{aligned}
$$

The expectation value of two bath operators defines the bath correlation function

$$
\mathcal{C}_{\alpha \beta}(\tau) \equiv\left\langle\boldsymbol{B}_{\alpha}(\tau) \boldsymbol{B}_{\beta}(0)\right\rangle,
$$

where bold symbols denote the interaction picture $\boldsymbol{B}_{\alpha}(\tau)$ $=e^{+\mathrm{i} \overline{\mathcal{H}}_{\mathrm{B}} \tau} B_{\alpha} e^{-\mathrm{i} \overline{\mathcal{H}}_{\mathrm{B}} \tau}$ and where the reservoir $\rho_{\mathrm{B}}=\rho_{\mathrm{B}}^{\mathrm{L}} \otimes \rho_{\mathrm{B}}^{\mathrm{R}} \otimes \rho_{\mathrm{B}}^{\mathrm{ph}}$ is a tensor product of thermalized states of left and right electronic leads and the phonon reservoir, respectively. This simple tensor-product approximation in the polaron-transformed frame does not hold in the original frame, where one obtains a displaced thermal phonon state depending on the electronic occupations, which is explicitly shown in Appendix C.

When the electronic reservoirs are weakly coupled and sufficiently Markovian (formalized by the condition $\left.\beta_{\alpha} \Gamma_{\alpha} \ll 1\right)$, perturbation theory in the electron-lead tunneling amplitudes $t_{k \sigma}$ and in $T_{\mathrm{c}}(\kappa-1)$, i.e., either in the asymmetry of the electron-phonon coupling or in $T_{\mathrm{c}}$ itself (for a continuum of phonon modes we just require a perturbative treatment in the $t_{k \sigma}$ ), leads to a Lindblad master equation, which appears particularly simple in the system energy eigenbasis. We label the eigenvectors of $\overline{\mathcal{H}}_{\mathrm{S}}$ as $|a\rangle \in\{|0\rangle,|-\rangle,|+\rangle,|2\rangle\}$, with system eigenenergies

$$
\begin{aligned}
& \varepsilon_{0} \equiv 0, \\
& \varepsilon_{-} \equiv \frac{1}{2}\left(\bar{\varepsilon}_{\mathrm{L}}+\bar{\varepsilon}_{\mathrm{R}}-\sqrt{\left(\bar{\varepsilon}_{\mathrm{L}}-\bar{\varepsilon}_{\mathrm{R}}\right)^{2}+4\left|\bar{T}_{\mathrm{c}}\right|^{2}}\right), \\
& \varepsilon_{+} \equiv \frac{1}{2}\left(\bar{\varepsilon}_{\mathrm{L}}+\bar{\varepsilon}_{\mathrm{R}}+\sqrt{\left(\bar{\varepsilon}_{\mathrm{L}}-\bar{\varepsilon}_{\mathrm{R}}\right)^{2}+4\left|\bar{T}_{\mathrm{c}}\right|^{2}}\right), \\
& \varepsilon_{2} \equiv \bar{\varepsilon}_{\mathrm{L}}+\bar{\varepsilon}_{\mathrm{R}}+\bar{U} .
\end{aligned}
$$

When the system spectrum is non-degenerate (more precisely, when the splitting $\varepsilon_{+}-\varepsilon_{-}$in $\overline{\mathcal{H}}_{\mathrm{S}}$ is much larger than the electronic tunneling amplitudes in the Hamiltonian), this will lead to a rate equation for the populations in the system energy eigenbasis

$$
\dot{\rho}_{a a}=+\sum_{b} \gamma_{a b, a b} \rho_{b b}-\left[\sum_{b} \gamma_{b a, b a}\right] \rho_{a a},
$$

where the positive rates ${ }^{76}$

$$
\gamma_{a b, a b}=\sum_{\alpha \beta} \gamma_{\alpha \beta}\left(\epsilon_{b}-\epsilon_{a}\right)\left\langle a\left|A_{\beta}\right| b\right\rangle\left\langle a\left|A_{\alpha}^{\dagger}\right| b\right\rangle^{*}
$$

are given by matrix elements of the system coupling operators in the energy eigenbasis and the Fourier transform of the reservoir correlation functions

$$
\gamma_{\alpha \beta}(\omega)=\int d t e^{+\mathrm{i} \omega t} \mathcal{C}_{\alpha \beta}(t)
$$

The coherences evolve independently from the populations. In particular, since in our model only coherences between states with the same charge may exist, we have

$$
\begin{aligned}
\dot{\rho}_{-+}= & -\mathrm{i}\left(E_{-}-E_{+}+\sigma_{--}-\sigma_{++}\right) \rho_{-+} \\
& +\left[\gamma_{--,++}-\frac{\gamma_{0-, 0-}+\gamma_{0+, 0+}+\gamma_{2-, 2-}+\gamma_{2+, 2+}}{2}\right. \\
& \left.-\frac{\gamma_{-+,-+}+\gamma_{+-,+-}}{2}\right] \rho_{-+},
\end{aligned}
$$

where $\sigma_{--}, \sigma_{++} \in \mathbb{R}$ describe a level-renormalization (Lambshift). We note that the rates $\gamma_{a b, a b}$ in Eq. (26) which describe electronic tunneling with phononic excitation between system and leads can be decomposed into interaction with left (L) and right $(\mathrm{R})$ bath

$$
\gamma_{a b, a b} \equiv \Gamma_{\mathrm{L}}^{a b}+\Gamma_{\mathrm{R}}^{a b},
$$

whereas the internal electronic transitions $\gamma_{-+,-+}$and $\gamma_{+-,+-}$ describing the relaxation from + to - or the excitation from - to + , respectively, only involve phonons

$$
\gamma_{-+,-+} \equiv \Gamma_{\mathrm{ph}}^{-+}, \quad \gamma_{+-,+-} \equiv \Gamma_{\mathrm{ph}}^{+-} .
$$

Furthermore, the matrix elements in the rates describing backward and forward processes triggered by the same reservoir are identical, such that local detailed balance is only induced by a corresponding Kubo-Martin-Schwinger (KMS)-type condition of the correlation functions. We discuss these for our system in Secs. III B and III C.

As a distinctive feature in comparison to a single quantum $\operatorname{dot}^{64}$ or to models without internal phonon-independent electronic tunneling, one now obtains phonon-modified internal transitions, and the corresponding rates between energy eigenstates $|-\rangle$ and $|+\rangle$ can be written as a quadratic form

$$
\begin{gathered}
\Gamma_{\mathrm{ph}}^{-+}=\left(A_{5}^{+-},\left(A_{5}^{-+}\right)^{*}\right) \underline{\gamma\left(\varepsilon_{+}-\varepsilon_{-}\right)}\left(\begin{array}{c}
\left(A_{5}^{+-}\right)^{*} \\
A_{5}^{-+}
\end{array}\right), \\
\Gamma_{\mathrm{ph}}^{+-}=\left(A_{5}^{-+},\left(A_{5}^{+-}\right)^{*}\right) \underline{\gamma\left(\varepsilon_{-}-\varepsilon_{+}\right)}\left(\begin{array}{c}
\left(A_{5}^{-+}\right)^{*} \\
A_{5}^{+-}
\end{array}\right),
\end{gathered}
$$

with the matrix $\gamma(\omega)$ being given by

$$
\underline{\gamma(\omega)}=\left(\begin{array}{ll}
\gamma_{56}(\omega) & \gamma_{55}(\omega) \\
\gamma_{66}(\omega) & \gamma_{65}(\omega)
\end{array}\right)
$$

It can be shown that this matrix is Hermitian and positive definite, such that we obtain true rates $\Gamma_{\mathrm{ph}}^{-+} \geq 0$ and $\Gamma_{\mathrm{ph}}^{+-} \geq 0$. Furthermore, we note that since the correlation functions contained in the matrix (30) obey KMS relations of the form $\gamma_{\alpha \beta}(-\omega)=\gamma_{\beta \alpha}(+\omega) e^{-\beta_{\mathrm{ph}} \omega}$ with inverse phonon reservoir temperature $\beta_{\mathrm{ph}}$ (compare Sec. III C), this implies for the ratio of rates $\frac{\Gamma_{\mathrm{ph}}^{+-}}{\Gamma_{\mathrm{ph}}^{-+}}=e^{-\beta_{\mathrm{ph}}\left(\varepsilon_{+}-\varepsilon_{-}\right)}$.

\section{B. Lead-phonon correlation function}

From Eq. (20), it follows that the four non-vanishing contributions associated with electronic jumps into or out of the system can be written in a product form of electronic and phononic contributions ${ }^{64}$

$$
\mathcal{C}_{\alpha \beta}(\tau)=\mathcal{C}_{\alpha \beta}^{\mathrm{el}}(\tau) \mathcal{C}_{\alpha \beta}^{\mathrm{ph}}(\tau)
$$


with the electronic parts being given by

$$
\begin{aligned}
& \mathcal{C}_{12}^{\mathrm{el}}(\tau)=\sum_{k}\left|t_{k \mathrm{~L}}\right|^{2} f_{\mathrm{L}}\left(\varepsilon_{k \mathrm{~L}}\right) e^{+\mathrm{i} \varepsilon_{k \mathrm{~L}} \tau}, \\
& \mathcal{C}_{21}^{\mathrm{el}}(\tau)=\sum_{k}\left|t_{k \mathrm{~L}}\right|^{2}\left[1-f_{\mathrm{L}}\left(\varepsilon_{k \mathrm{~L}}\right)\right] e^{-\mathrm{i} \varepsilon_{k \mathrm{~L}} \tau}, \\
& \mathcal{C}_{34}^{\mathrm{el}}(\tau)=\sum_{k}^{k}\left|t_{k \mathrm{R}}\right|^{2} f_{\mathrm{R}}\left(\varepsilon_{k \mathrm{R}}\right) e^{+\mathrm{i} \varepsilon_{k \mathrm{R}} \tau}, \\
& \mathcal{C}_{43}^{\mathrm{e}}(\tau)=\sum_{k}\left|t_{k \mathrm{R}}\right|^{2}\left[1-f_{\mathrm{R}}\left(\varepsilon_{k \mathrm{R}}\right)\right] e^{-\mathrm{i} \varepsilon_{k \mathrm{R}} \tau} .
\end{aligned}
$$

Here, we have introduced the Fermi function $f_{\sigma}(\omega) \equiv\left[e^{\beta_{\sigma}\left(\omega-\mu_{\sigma}\right)}+1\right]^{-1}$ of lead $\sigma$ with inverse temperature $\beta_{\sigma}$ and chemical potential $\mu_{\sigma}$. The tunneling amplitudes $t_{k \sigma}$ lead to effective tunneling rates $\Gamma_{\sigma}(\omega)$ $\equiv 2 \pi \sum_{k}\left|t_{k \sigma}\right|^{2} \delta\left(\omega-\varepsilon_{k \sigma}\right)$, which can be used to convert the above summations into integrals. Later-on, we will parametrize them with a Lorentzian distribution ${ }^{78}$

$$
\Gamma_{\sigma}(\omega) \equiv \frac{\Gamma_{\sigma} \delta_{\sigma}^{2}}{\omega^{2}+\delta_{\sigma}^{2}}
$$

yielding a representation in terms of hypergeometric functions for $C_{\alpha \beta}^{\mathrm{el}}(\tau)$, which we omit here for brevity. For completeness, we note that the separate Fourier transforms of the electronic parts $\gamma_{\alpha \beta}^{\mathrm{el}}(\omega)=\int \mathcal{C}_{\alpha \beta}^{\mathrm{el}}(\tau) e^{+\mathrm{i} \omega \tau} d \tau$,

$$
\begin{aligned}
& \gamma_{12}^{\mathrm{el}}(\omega)=\Gamma_{\mathrm{L}}(-\omega) f_{\mathrm{L}}(-\omega), \\
& \gamma_{21}^{\mathrm{el}}(\omega)=\Gamma_{\mathrm{L}}(+\omega)\left[1-f_{\mathrm{L}}(+\omega)\right], \\
& \gamma_{34}^{\mathrm{el}}(\omega)=\Gamma_{\mathrm{R}}(-\omega) f_{\mathrm{R}}(-\omega), \\
& \gamma_{43}^{\mathrm{el}}(\omega)=\Gamma_{\mathrm{R}}(+\omega)\left[1-f_{\mathrm{R}}(+\omega)\right],
\end{aligned}
$$

obey-since $f_{\sigma}(\omega)=e^{-\beta_{\sigma}\left(\omega-\mu_{\sigma}\right)}\left[1-f_{\sigma}(\omega)\right]$-the KMS-type relations

$$
\begin{aligned}
& \gamma_{12}^{\mathrm{el}}(-\omega)=e^{-\beta_{\mathrm{L}}\left(\omega-\mu_{\mathrm{L}}\right)} \gamma_{21}^{\mathrm{el}}(+\omega), \\
& \gamma_{34}^{\mathrm{el}}(-\omega)=e^{-\beta_{\mathrm{R}}\left(\omega-\mu_{\mathrm{R}}\right)} \gamma_{43}^{\mathrm{el}}(+\omega) .
\end{aligned}
$$

The phonon contribution to the correlation function depends only on the terminal across which the electron jumps but not on the jump direction, i.e., we have $\mathcal{C}_{12}^{\mathrm{ph}}(\tau)=\mathcal{C}_{21}^{\mathrm{ph}}(\tau)$ $\equiv \mathcal{C}_{\mathrm{L}}^{\mathrm{ph}}(\tau)$ and $\mathcal{C}_{34}^{\mathrm{ph}}(\tau)=\mathcal{C}_{43}^{\mathrm{ph}}(\tau) \equiv \mathcal{C}_{\mathrm{R}}^{\mathrm{ph}}(\tau)$. Using the BakerCampbell-Hausdorff (BCH) formula, the phonon contribution explicitly computes to (see Appendix D 1),

$$
\mathcal{C}_{\sigma}^{\mathrm{ph}}(\tau)=e^{-K_{\sigma}(0)+K_{\sigma}(\tau)},
$$

with the abbreviation in the exponent

$$
\begin{aligned}
K_{\sigma}(\tau)= & \sum_{q} \frac{\left|h_{q \sigma}\right|^{2}}{\omega_{q}^{2}} \\
& \times\left\{n_{\mathrm{B}}\left(\omega_{q}\right) e^{+\mathrm{i} \omega_{q} \tau}+\left[n_{\mathrm{B}}\left(\omega_{q}\right)+1\right] e^{-\mathrm{i} \omega_{q} \tau}\right\} .
\end{aligned}
$$

It is easy to show that $K_{\sigma}(\tau)=K_{\sigma}\left(-\tau-\mathrm{i} \beta_{\mathrm{ph}}\right)$ holds, which transfers to the KMS condition for the phonon contribution to the correlation function

$$
\mathcal{C}_{\sigma}^{\mathrm{ph}}(\tau)=\mathcal{C}_{\sigma}^{\mathrm{ph}}\left(-\tau-\mathrm{i} \beta_{\mathrm{ph}}\right)
$$

The nature of the phonon contributions can now be quite distinct depending on whether one has a discrete (e.g., just a single mode) or continuous spectrum of phonon frequencies. In the continuum case, we can convert the sum in the exponent into an integral. Then, the phonon absorption emission amplitudes enter the corresponding rate as $\mathcal{J}_{\sigma}(\omega)$ $\equiv \sum_{q}\left|h_{q \sigma}\right|^{2} \delta\left(\omega-\omega_{q}\right)$, where $\mathcal{J}_{\mathrm{L}}(\omega)$ and $\mathcal{J}_{\mathrm{R}}(\omega)$ will be parametrized by a continuous function. For example, using the super-ohmic parameterization with exponential infrared cutoff at $\omega_{\mathrm{c}}^{\sigma}$ (we choose a super-ohmic representation to enable a Markovian description of the internal jumps in Sec. III C) and coupling strength $J_{\sigma}$, i.e.,

$$
\mathcal{J}_{\sigma}(\omega) \equiv J_{\sigma} \omega^{3} e^{-\frac{\omega}{\omega_{\mathrm{c}}^{\sigma}}}
$$

we obtain for the integrals in the exponent

$$
\begin{aligned}
K_{\sigma}(\tau) & =\int_{0}^{\infty} \frac{\mathcal{J}_{\sigma}(\omega)}{\omega^{2}}\left[n_{\mathrm{B}}(\omega) e^{+\mathrm{i} \omega \tau}+\left[1+n_{\mathrm{B}}(\omega)\right] e^{-\mathrm{i} \omega \tau}\right] d \omega \\
& =\frac{2 J_{\sigma}}{\beta^{2}} \mathfrak{R}\left\{\Psi^{\prime}\left(\frac{1+\mathrm{i} \tau \omega_{\mathrm{c}}^{\sigma}}{\beta_{\mathrm{ph}} \omega_{\mathrm{c}}^{\sigma}}\right)\right\}-\frac{J_{\sigma}\left(\omega_{\mathrm{c}}^{\sigma}\right)^{2}}{\left(1-\mathrm{i} \tau \omega_{\mathrm{c}}^{\sigma}\right)^{2}},
\end{aligned}
$$

where $\Psi^{\prime}(x)$ denotes the derivative of the polygamma function $\Psi(x)=\Gamma^{\prime}(x) / \Gamma(x)$. With the same super-ohmic spectral density, the renormalized on-site energies and Coulomb shift read explicitly

$$
\begin{aligned}
\bar{\varepsilon}_{\sigma} & =\varepsilon_{\sigma}-2 J_{\sigma}\left(\omega_{\mathrm{c}}^{\sigma}\right)^{3}, \\
\bar{U} & =U+\sum_{q} \frac{\left|h_{q \mathrm{~L}}-h_{q \mathrm{R}}\right|^{2}-\left|h_{q \mathrm{~L}}\right|^{2}-\left|h_{q \mathrm{R}}\right|^{2}}{\omega_{q}} \\
& =U+2 J_{0}\left(\omega_{\mathrm{c}}^{0}\right)^{3}-2 J_{\mathrm{L}}\left(\omega_{\mathrm{c}}^{\mathrm{L}}\right)^{3}-2 J_{\mathrm{R}}\left(\omega_{\mathrm{c}}^{\mathrm{R}}\right)^{3} .
\end{aligned}
$$

We note here that since $K_{\sigma}(\tau)$ in Eq. (40) decays to zero for large $\tau$, the phonon correlation function $C_{\sigma}^{\mathrm{ph}}(\tau)$ may remain finite for large $\tau$. Thanks to the influence of the electronic contributions the total correlation function will still decay, such that a Markovian approach is applicable. In this case, we technically define separate Fourier transforms of the phonon contributions by

$$
\begin{aligned}
\gamma_{\sigma}^{\mathrm{ph}}(\omega)= & \int\left[\mathcal{C}_{\sigma}^{\mathrm{ph}}(\tau)-\mathcal{C}_{\sigma}^{\mathrm{ph}}(\infty)\right] e^{+\mathrm{i} \omega \tau} d \tau \\
& +2 \pi \mathcal{C}_{\sigma}^{\mathrm{ph}}(\infty) \delta(\omega) .
\end{aligned}
$$

Since the dressed correlation functions are given by products of electronic and phononic contributions in the time domain, the separate KMS relations (35) and (38) do not directly transfer in non-equilibrium setups. However, we can use our previous result (see appendix of Ref. 64) that these correlation functions can be written conditioned upon the net number $\boldsymbol{n}=\left(n_{1}, \ldots, n_{Q}\right)$ of emitted phonons into the different reservoir modes $\left(n_{q}<0\right.$ implies absorption from the phonon reservoir). Formally, one has $\gamma_{\alpha \beta}(\omega)=\sum_{n} \gamma_{\alpha \beta, n}(\omega)$, where the separate contributions are given by $\left(\boldsymbol{\Omega}=\left(\omega_{1}, \ldots, \omega_{Q}\right)\right)$,

$$
\begin{aligned}
\gamma_{\alpha \beta, n}(\omega)= & \gamma_{\alpha \beta}^{\mathrm{el}}(\omega-\boldsymbol{n} \cdot \boldsymbol{\Omega}) \prod_{q} e^{-\frac{\left|h_{q}\right|^{2}}{\omega_{q}^{2}}\left(1+2 n_{\mathrm{B}}^{q}\right)} \times\left(\frac{1+n_{\mathrm{B}}^{q}}{n_{\mathrm{B}}^{q}}\right)^{n_{q} / 2} \\
& \times \mathcal{J}_{n_{q}}\left(2 \frac{\left|h_{q}\right|^{2}}{\omega_{q}^{2}} \sqrt{n_{\mathrm{B}}^{q}\left(1+n_{\mathrm{B}}^{q}\right)}\right)
\end{aligned}
$$

with $\mathcal{J}_{n}(x) \equiv \sum_{k=0}^{\infty}\left\{(-1)^{k} / k ! \Gamma[k+n+1]\right\}(x / 2)^{2 k+n}$ being the modified Bessel function of the first kind and $\Gamma[x] \equiv \int_{0}^{\infty} t^{x-1} e^{-t}$ $\mathrm{d} t$ being the Gamma-function. We note that when the electronic Fourier transforms are flat $\gamma_{\alpha \beta}^{\mathrm{el}}(\omega-\boldsymbol{n} \cdot \mathbf{\Omega}) \rightarrow \bar{\gamma}_{\alpha \beta}^{\mathrm{el}}$, the 
normalization of the phonon contribution implies that the Fourier transform of the combined correlation function is also flat, $\sum_{n} \gamma_{\alpha \beta, n}(\omega) \rightarrow \bar{\gamma}_{\alpha \beta}^{\text {el }}$. This implies that in the electronic wide-band $\left(\delta_{\sigma} \rightarrow \infty\right)$ plus the infinite bias $\left(f_{\mathrm{L}}(\omega) \rightarrow 1\right.$ and $\left.f_{\mathrm{R}}(\omega) \rightarrow 0\right)$ limits the phonons will have no effect on the dotlead correlation functions.

Importantly, we note that even for different temperatures, these obey the KMS-type relation

$$
\begin{aligned}
\gamma_{12,+n_{\mathbf{L}}}(-\omega)= & e^{-\beta_{\mathrm{L}}\left(\omega-\mu_{\mathrm{L}}+\boldsymbol{n}_{\mathbf{L}} \cdot \mathbf{\Omega}\right)} e^{+\beta_{\mathrm{ph}} \boldsymbol{n}_{\mathbf{L}} \cdot \mathbf{\Omega}} \\
& \times \gamma_{21,-\boldsymbol{n}_{\mathbf{L}}}(+\omega), \\
\gamma_{34,+\boldsymbol{n}_{\mathbf{R}}}(-\omega)= & e^{-\beta_{\mathrm{R}}\left(\omega-\mu_{\mathrm{R}}+\boldsymbol{n}_{\mathbf{R}} \cdot \mathbf{\Omega}\right)} e^{+\beta_{\mathrm{ph}} \boldsymbol{n}_{\mathbf{R}} \cdot \mathbf{\Omega}} \\
& \times \gamma_{43,-\boldsymbol{n}_{\mathbf{R}}}(+\omega) .
\end{aligned}
$$

We see that the conventional KMS relation is reproduced when phonon and electronic temperatures are equal.

\section{Interdot-phonon correlation function}

To evaluate the transitions between the states $|-\rangle \leftrightarrow|+\rangle$, we have to evaluate the correlation functions

$$
\begin{aligned}
& \mathcal{C}_{55}(\tau)=\left\langle e^{-\mathcal{B}_{\mathrm{L}}(\tau)} e^{+\mathcal{B}_{\mathrm{R}}(\tau)} e^{-\mathcal{B}_{\mathrm{L}}} e^{+\mathcal{B}_{\mathrm{R}}}\right\rangle-\kappa^{2}, \\
& \mathcal{C}_{66}(\tau)=\left\langle e^{-\mathcal{B}_{\mathrm{R}}(\tau)} e^{+\mathcal{B}_{\mathrm{L}}(\tau)} e^{-\mathcal{B}_{\mathrm{R}}} e^{+\mathcal{B}_{\mathrm{L}}}\right\rangle-\left(\kappa^{*}\right)^{2}, \\
& \mathcal{C}_{56}(\tau)=\left\langle e^{-\mathcal{B}_{\mathrm{L}}(\tau)} e^{+\mathcal{B}_{\mathrm{R}}(\tau)} e^{-\mathcal{B}_{\mathrm{R}}} e^{+\mathcal{B}_{\mathrm{L}}}\right\rangle-|\kappa|^{2}, \\
& \mathcal{C}_{65}(\tau)=\left\langle e^{-\mathcal{B}_{\mathrm{R}}(\tau)} e^{+\mathcal{B}_{\mathrm{L}}(\tau)} e^{-\mathcal{B}_{\mathrm{L}}} e^{+\mathcal{B}_{\mathrm{R}}}\right\rangle-|\kappa|^{2},
\end{aligned}
$$

where we have used that $\kappa=\left\langle e^{-\mathcal{B}_{\mathrm{L}}} e^{+\mathcal{B}_{\mathrm{R}}}\right\rangle=\left\langle e^{-\mathcal{B}_{\mathrm{L}}(\tau)} e^{+\mathcal{B}_{\mathrm{R}}(\tau)}\right\rangle$ is inert with respect to transformations into the interaction picture. For the first bath correlation functions we obtain (see Appendix D 2),

$$
\mathcal{C}_{55}(\tau)=\kappa^{2}\left[e^{-K(\tau)}-1\right]
$$

where-in analogy to Eq. (37)—we have

$$
\begin{aligned}
K(\tau)= & \sum_{q} \frac{\left|h_{q \mathrm{~L}}-h_{q \mathrm{R}}\right|^{2}}{\omega_{q}^{2}} \\
& \times\left\{n_{\mathrm{B}}\left(\omega_{q}\right) e^{+\mathrm{i} \omega_{q} \tau}+\left[n_{\mathrm{B}}\left(\omega_{q}\right)+1\right] e^{-\mathrm{i} \omega_{q} \tau}\right\} .
\end{aligned}
$$

We note that for large times the correlation function vanishes for a continuum of phonon modes, facilitating a Markovian description. Two further correlation functions can be similarly evaluated

$$
\mathcal{C}_{66}(\tau)=\left(\kappa^{*}\right)^{2}\left[e^{-K(\tau)}-1\right]=\mathcal{C}_{55}^{*}(-\tau)
$$

where the latter equality can be easily seen by direct comparison. For the third correlation function, we find

$$
\mathcal{C}_{56}(\tau)=|\kappa|^{2}\left[e^{K(\tau)}-1\right] .
$$

It can be easily seen that $\mathcal{C}_{56}(t) \hat{=} \mathcal{C}_{65}(t)$. Furthermore, we note that

$$
\begin{aligned}
\kappa^{2} & =e^{-K(0)} e^{+2 \mathrm{i} \Phi}, \quad\left(\kappa^{*}\right)^{2}=e^{-K(0)} e^{-2 \mathrm{i} \Phi}, \\
|\kappa|^{2} & =e^{-K(0)} .
\end{aligned}
$$

From $K(-\tau)=K^{*}(+\tau)$ we conclude that the Fourier transform matrix of these correlation functions (30) is Hermitian. It can be expressed by the two real-valued functions

$$
\gamma_{ \pm}(\omega)=\int\left(e^{ \pm K(\tau)}-1\right) e^{+\mathrm{i} \omega \tau} d \tau
$$

and will be positive definite at frequency $\omega$ when $\gamma_{-}(\omega)$ $<\gamma_{+}(\omega)$ or, equivalently, when $\gamma_{+}^{2}(\omega)-\gamma_{-}^{2}(\omega)=\left[\gamma_{+}(\omega)\right.$ $\left.-\gamma_{-}(\omega)\right]\left[\gamma_{+}(\omega)+\gamma_{-}(\omega)\right]>0$. The interdot phonon correlation functions obey KMS relations of the type (for $\alpha, \beta \in\{5,6\}$ ),

$$
C_{\alpha \beta}(\tau)=C_{\beta \alpha}\left(-\tau-\mathrm{i} \beta_{\mathrm{ph}}\right),
$$

which follow from the definition of $K(\tau)$. For their Fourier transforms this implies $\gamma_{\alpha \beta}(-\omega)=\gamma_{\beta \alpha}(+\omega) e^{-\beta \omega}$.

Finally, we note that this approach is valid for coupling to a continuum of phonon modes. A finite number of phonon modes would in general not lead to a decay of the interdot correlation functions $\mathcal{C}_{55}(\tau), \mathcal{C}_{56}(\tau), \mathcal{C}_{65}(\tau)$, and $\mathcal{C}_{66}(\tau)$, thus prohibiting a Markovian description. Furthermore, the electronic tunneling Hamiltonian $\overline{\mathcal{H}}_{\mathrm{V}}$ and the inter-dot tunneling Hamiltonian $\overline{\mathcal{H}}_{\mathrm{T}}$ must be small in the polaron frame. The first condition is consistent with a perturbative treatment of electron-lead tunneling amplitudes, whereas the second condition can be fulfilled by choosing either nearly symmetric electron-phonon couplings left and right, i.e., $h_{q \mathrm{~L}} \approx h_{q} \approx h_{q \mathrm{R}}$ or by treating $T_{\mathrm{c}}$ also perturbatively. If the electron-phonon coupling is exactly symmetric, also finite phonon modes can be treated with the approach.

\section{Numerical phonon correlation function}

In case of a continuous phonon spectrum, the Fourier transforms of the phonon correlation functions associated with external-compare Eq. (36)—and internal-compare Eqs. (46), (48), and (49)—electronic jumps cannot be obtained analytically in closed form. This complicates the calculation of the full transition rates whenever one is also interested in the heat exchanged with the phonon reservoir, as this requires evaluation of a convolution integral, where the phonon contribution to the integrand is itself a numerical Fourier integral. Here, we therefore aim to represent the Fourier-transform of the phonon contribution in a semi-exact fashion, respecting the thermodynamic KMS relations. For this, we note that the Gaussian

$$
\gamma_{\mathrm{ph}}^{\mathrm{fit}}(\omega)=a e^{-\frac{\left(\omega-\beta_{\mathrm{ph}} b / 4\right)^{2}}{b}}
$$

obeys for all fit parameters $a$ and $b$ and frequencies $\omega$; the KMS relation $\frac{\gamma_{\mathrm{ph}}^{\text {fit }}(+\omega)}{\gamma_{\mathrm{ph}}^{\text {fit }}(-\omega)}=e^{\beta_{\mathrm{ph}} \omega}$, where $\beta_{\mathrm{ph}}$ denotes the inverse phonon temperature. Naturally, by fitting the phonon correlation functions, e.g., with multiple such Gaussian functions, one would obtain a thermodynamic correct representation of the phonon correlation function. Here, however, we are rather interested in thermodynamic principles and just use a single Gaussian function, where we fix the fit parameters by crudely matching $C_{\mathrm{ph}}^{\mathrm{fit}}(0)$ and $\int C_{\mathrm{ph}}^{\mathrm{fit}}(\tau) d \tau$ with the true values of the correlation function. We note that both $C_{\mathrm{ph}}(0)$ and $\int C_{\mathrm{ph}}(\tau) d \tau$ are always real-valued, such that the Fourier transform of the Gaussian approximation does not only obey the KMS condition but is also always positive. 


\section{SYMMETRIES IN THE FULL COUNTING STATISTICS}

To deduce the counting statistics not only of electrons but also of the phonons, it would be necessary to identify the phonons emitted or absorbed with every electronic jump. However, here we are rather interested in the energy that by such processes is emitted into or absorbed from the phonon reservoir. For internal electronic transitions, the energy exchange follows directly from the change in the system state. In contrast, for transitions involving an electronic jump across the left or right terminal, one has to identify the separate phononic contributions to correctly partition the electronic and phononic contributions to the exchanged energy.

To identify a minimal set of transitions that has to be monitored for energy and particle exchange, we first consider the entropy production $\dot{S}_{\mathrm{i}}$ in the system, which at steady state must be balanced by the entropy flow $\dot{S}_{\mathrm{e}}$ from the electronic and phononic terminals ${ }^{68}$

$$
\begin{aligned}
\dot{S}_{\mathrm{i}} & =-\dot{S}_{\mathrm{e}}=-\sum_{v} \beta_{v} \dot{Q}_{v} \\
& =-\beta_{\mathrm{L}}\left(I_{E}^{\mathrm{L}}-\mu_{\mathrm{L}} I_{M}^{\mathrm{L}}\right)-\beta_{\mathrm{R}}\left(I_{E}^{\mathrm{R}}-\mu_{\mathrm{R}} I_{M}^{\mathrm{R}}\right)-\beta_{\mathrm{ph}} I_{E}^{\mathrm{ph}},
\end{aligned}
$$

where $I_{E}^{v}, I_{M}^{v}$, and $\dot{Q}_{v}$ denote the energy, matter, and heat currents from terminal $v$ into the system, respectively. Using the conservation laws for energy and matter

$$
I_{\mathrm{E}}^{\mathrm{L}}+I_{\mathrm{E}}^{\mathrm{R}}+I_{\mathrm{E}}^{\mathrm{ph}}=0, \quad I_{\mathrm{M}}^{\mathrm{L}}+I_{\mathrm{M}}^{\mathrm{R}}=0,
$$

we can eliminate two currents.

We choose to monitor the number of electrons entering the system from the left lead $I_{\mathrm{M}}^{(\mathrm{L})}$, the energy that is transferred from the left lead into the system $I_{\mathrm{E}}^{(\mathrm{L})}$, and the energy that is transferred from the phonon reservoir into the system $I_{\mathrm{E}}^{(\mathrm{ph})}$. In terms of these quantities, the entropy production becomes

$$
\dot{S}_{\mathrm{i}}=\left(\beta_{\mathrm{R}}-\beta_{\mathrm{L}}\right) I_{\mathrm{E}}^{\mathrm{L}}+\left(\beta_{\mathrm{L}} \mu_{\mathrm{L}}-\beta_{\mathrm{R}} \mu_{\mathrm{R}}\right) I_{\mathrm{M}}^{\mathrm{L}}+\left(\beta_{\mathrm{R}}-\beta_{\mathrm{ph}}\right) I_{\mathrm{E}}^{\mathrm{ph}},
$$

which is decomposable into affinities and fluxes. When we further assume that the electronic temperatures of both leads are the same $\beta_{\mathrm{L}}=\beta_{\mathrm{R}}=\beta_{\mathrm{el}}$, the entropy production can even be expressed with only two affinities and two fluxes

$$
\dot{S}_{\mathrm{i}}=\beta_{\mathrm{el}}\left(\mu_{\mathrm{L}}-\mu_{\mathrm{R}}\right) I_{\mathrm{M}}^{\mathrm{L}}+\left(\beta_{\mathrm{el}}-\beta_{\mathrm{ph}}\right) I_{\mathrm{E}}^{\mathrm{ph}} .
$$

Formally, the statistics of energy and matter transfers can be extracted by complementing the off-diagonal entries in the Liouvillian that describe the individual jump processes with counting fields. For the electronic hopping this is fairly standard and straightforward to do. It becomes a bit more involved, however, when one is interested in the statistics of energy exchanges: for the internal jumps—see Eq. (28)—the energy counting field $\phi$ is multiplied by the complete energy that is exchanged with the phonon reservoir

$$
\begin{aligned}
\Gamma_{\mathrm{ph}}^{-+} & \rightarrow \Gamma_{\mathrm{ph}}^{-+} e^{-\mathrm{i} \phi\left(\varepsilon_{+}-\varepsilon_{-}\right)}, \\
\Gamma_{\mathrm{ph}}^{+-} & \rightarrow \Gamma_{\mathrm{ph}}^{+-} e^{+\mathrm{i} \phi\left(\varepsilon_{+}-\varepsilon_{-}\right)} .
\end{aligned}
$$

For the electronic jumps between system and both leads we however have to partition the emitted or absorbed energy into contributions from the electronic and phononic reservoirs, which first requires to decompose the transitions into different phonon contributions. Assuming, for example, a discrete phonon spectrum, we have

$$
\Gamma_{\sigma}^{a b}=\sum_{n} \Gamma_{\sigma}^{a b, n},
$$

where $\Gamma_{\sigma}^{a b, n}$ describes a transition from energy eigenstate $j$ to $\boldsymbol{i}$ together with the emission of $\boldsymbol{n}$ phonons into the different phonon reservoir modes and an electronic jump to or from lead $\sigma \in\{\mathrm{L}, \mathrm{R}\}$-see Eq. (27). For a continuous phonon spectrum (which we will not discuss explicitly) we could use the convolution theorem to arrive at a similar decomposition, $\Gamma_{\sigma}^{a b}=\int \Gamma_{\sigma}^{a b}(\omega) d \omega$, where $\Gamma_{\sigma}^{a b}(\omega)$ describes a transition from energy eigenstate $b$ to $a$ together with the emission of energy $\omega$ into the phonon reservoir and an electronic jump to or from lead $\sigma \in\{\mathrm{L}, \mathrm{R}\}$. This then implies the counting field replacements for the off-diagonal matrix elements in the Liouvillian

$$
\begin{aligned}
\Gamma_{\mathrm{L}}^{a b, n} & \rightarrow \Gamma_{\mathrm{L}}^{a b, \boldsymbol{n}} e^{+\mathrm{i} \chi\left(n_{a}-n_{b}\right)} e^{+\mathrm{i} \xi\left(\epsilon_{a}-\epsilon_{b}+n \cdot \boldsymbol{\Omega}\right)} e^{-\mathrm{i} \phi \boldsymbol{n} \cdot \mathbf{\Omega}}, \\
\Gamma_{\mathrm{R}}^{i j, n} & \rightarrow \Gamma_{\mathrm{R}}^{a b, n} e^{-\mathrm{i} \phi \boldsymbol{n} \cdot \mathbf{\Omega},}
\end{aligned}
$$

where $n_{a} \in\{0,1,2\}$ denotes the number of electrons in energy eigenstate $a$. Thus, the Liouvillian is now dependent on the particle counting field $\chi$, the electronic energy counting field $\xi$, and the phonon energy counting field $\phi$, which we may for brevity combine in a vector $\chi=(\chi, \xi, \phi)$. The characteristic polynomial $\mathcal{D}(\chi)=|\mathcal{L}(\chi)-\lambda \mathbf{1}|$ of the now counting-field dependent Liouvillian formally equates to

$$
\begin{aligned}
\mathcal{D}= & {\left[\mathcal{L}_{11}-\lambda\right]\left[\mathcal{L}_{22}-\lambda\right]\left[\mathcal{L}_{33}-\lambda\right]\left[\mathcal{L}_{44}-\lambda\right]-\left[\mathcal{L}_{11}-\lambda\right]\left[\mathcal{L}_{22}-\lambda\right] \mathcal{L}_{34} \mathcal{L}_{43}-\left[\mathcal{L}_{11}-\lambda\right]\left[\mathcal{L}_{33}-\lambda\right] \mathcal{L}_{24} \mathcal{L}_{42} } \\
& -\left[\mathcal{L}_{11}-\lambda\right]\left[\mathcal{L}_{44}-\lambda\right] \mathcal{L}_{23} \mathcal{L}_{32}-\left[\mathcal{L}_{22}-\lambda\right]\left[\mathcal{L}_{44}-\lambda\right] \mathcal{L}_{13} \mathcal{L}_{31}-\left[\mathcal{L}_{33}-\lambda\right]\left[\mathcal{L}_{44}-\lambda\right] \mathcal{L}_{12} \mathcal{L}_{21} \\
& +\left[\mathcal{L}_{11}-\lambda\right]\left[\mathcal{L}_{23} \mathcal{L}_{34} \mathcal{L}_{42}+\mathcal{L}_{24} \mathcal{L}_{43} \mathcal{L}_{32}\right]+\left[\mathcal{L}_{44}-\lambda\right]\left[\mathcal{L}_{12} \mathcal{L}_{23} \mathcal{L}_{31}+\mathcal{L}_{13} \mathcal{L}_{32} \mathcal{L}_{21}\right]+\mathcal{L}_{12} \mathcal{L}_{21} \mathcal{L}_{34} \mathcal{L}_{43}+\mathcal{L}_{13} \mathcal{L}_{31} \mathcal{L}_{24} \mathcal{L}_{42} \\
& -\mathcal{L}_{12} \mathcal{L}_{24} \mathcal{L}_{43} \mathcal{L}_{31}-\mathcal{L}_{13} \mathcal{L}_{34} \mathcal{L}_{42} \mathcal{L}_{21}
\end{aligned}
$$

where it should be kept in mind that the counting fields only occur in the off-diagonal $\left(\mathcal{L}_{i \neq j}\right)$ contributions. With the relations $(\sigma \in\{\mathrm{L}, \mathrm{R}\})$,

$$
\begin{aligned}
& \frac{\Gamma_{\mathrm{ph}}^{-+}}{\Gamma_{\mathrm{ph}}^{+-}}=e^{+\beta_{\mathrm{ph}}\left(\varepsilon_{+}-\varepsilon_{-}\right)}, \quad \frac{\Gamma_{\sigma}^{0-,-\boldsymbol{n}}}{\Gamma_{\sigma}^{-0,+\boldsymbol{n}}}=e^{+\beta_{\sigma}\left(\varepsilon_{-}-\varepsilon_{0}-\mu_{\sigma}+\boldsymbol{n} \cdot \mathbf{\Omega}\right)} e^{-\beta_{\mathrm{ph}} \boldsymbol{n} \cdot \mathbf{\Omega}}, \quad \frac{\Gamma_{\sigma}^{0+,-\boldsymbol{n}}}{\Gamma_{\sigma}^{+0,+\boldsymbol{n}}}=e^{+\beta_{\sigma}\left(\varepsilon_{+}-\varepsilon_{0}-\mu_{\sigma}+\boldsymbol{n} \cdot \mathbf{\Omega}\right)} e^{-\beta_{\mathrm{ph}} \boldsymbol{n} \cdot \mathbf{\Omega}}, \\
& \frac{\Gamma_{\sigma}^{-2,-n}}{\Gamma_{\sigma}^{2-,+n}}=e^{+\beta_{\sigma}\left(\varepsilon_{2}-\varepsilon_{-}-\mu_{\sigma}+n \cdot \mathbf{\Omega}\right)} e^{-\beta_{\mathrm{ph}} n \cdot \mathbf{\Omega}}, \quad \frac{\Gamma_{\sigma}^{+2,-n}}{\Gamma_{\sigma}^{2+,+n}}=e^{+\beta_{\sigma}\left(\varepsilon_{2}-\varepsilon_{+}-\mu_{\sigma}+n \cdot \mathbf{\Omega}\right)} e^{-\beta_{\mathrm{ph}} n \cdot \mathbf{\Omega}},
\end{aligned}
$$


one can show (compare Appendix E) that the characteristic polynomial stays invariant under the replacements

$$
\begin{aligned}
& -\chi \rightarrow+\chi+\mathrm{i}\left(\beta_{\mathrm{L}} \mu_{\mathrm{L}}-\beta_{\mathrm{R}} \mu_{\mathrm{R}}\right), \\
& -\xi \rightarrow+\xi+\mathrm{i}\left(\beta_{\mathrm{R}}-\beta_{\mathrm{L}}\right), \\
& -\phi \rightarrow+\phi+\mathrm{i}\left(\beta_{\mathrm{R}}-\beta_{\mathrm{ph}}\right),
\end{aligned}
$$

where we recover the affinities in Eq. (56). This symmetry transfers to the long-term cumulant-generating function, and thus, the steady state fluctuation theorem for entropy production reads

$$
\lim _{t \rightarrow \infty} \frac{P_{+n_{\mathrm{L}},+e_{\mathrm{L}},+e_{\mathrm{ph}}}(t)}{P_{-n_{\mathrm{L}},-e_{\mathrm{L}},-e_{\mathrm{ph}}}(t)}=e^{\mathbf{n} \Delta},
$$

with $\boldsymbol{n} \equiv\left(n_{\mathrm{L}}, e_{\mathrm{L}}, e_{\mathrm{ph}}\right)^{\mathrm{T}}$ and $\boldsymbol{\Delta}=\left(\beta_{\mathrm{L}} \mu_{\mathrm{L}}-\beta_{\mathrm{R}} \mu_{\mathrm{R}}, \beta_{\mathrm{R}}-\beta_{\mathrm{L}}, \beta_{\mathrm{R}}\right.$ $\left.-\beta_{\mathrm{ph}}\right)^{\mathrm{T}}$. Due to the similar three-terminal setup, the same fluctuation theorem can be obtained for the single electron transistor. ${ }^{64}$

\section{RESULTS}

The implications of the resulting master equation are of course manifold. Below, we present a selection of the most interesting phonon-induced features. For simplicity, we will discuss the case of symmetric couplings $h_{q \mathrm{~L}}=h_{q \mathrm{R}}$ here.

\section{A. Electronic current versus internal bias}

We compute the electronic matter current for coupling to a single phonon mode at frequency $\Omega$ and also for coupling to a continuum of phonons. Figure 2 shows the electronic current at infinite external bias $\left(f_{\mathrm{L}} \rightarrow 1, f_{\mathrm{R}} \rightarrow 0\right)$ but finite bandwidths as a function of the internal bias $\Delta \varepsilon \equiv \varepsilon_{\mathrm{L}}-\varepsilon_{\mathrm{R}}$, which we define symmetrically with $\varepsilon_{\mathrm{L}} \equiv+\Delta \varepsilon / 2$ and $\varepsilon_{\mathrm{R}} \equiv-\Delta \varepsilon / 2$. We note that due to the finite $T_{\mathrm{c}}$, the system spectrum remains in the non-degenerate regime also when $\varepsilon_{L}=\varepsilon_{R}$. The study of such currents is very common in theoretical ${ }^{79-82}$ studies as they reveal many internal details of the transport setup. In Fig. 2, the black curve shows the pure electronic current without phonons $\left(h_{q \mathrm{~L}}=h_{q \mathrm{R}}=0\right)$ far away from the wide-band $\operatorname{limit}\left(\delta_{\mathrm{L}} / \Omega=\delta_{\mathrm{L}} / \Omega=0.1\right)$. Here, two electronic resonances at $\pm\left(\varepsilon_{2}-\varepsilon_{-}\right) / \Omega= \pm 10$ become visible. The Lorentzian shape of the graph is characteristic for such models and stems from the matrix elements in front of the rates. For the colored curves, we increase the electron-phonon coupling $\left(h_{q \mathrm{~L}}=h_{q \mathrm{R}}=h_{q}\right)$ at large phonon bath temperature $\beta_{\mathrm{ph}} \Omega=0.1$ (due to the infinitebias assumption, the electronic temperature does not enter). Due to the coupling to a single phonon mode, we see additional side peaks appearing at $\Delta \varepsilon=2 n \Omega$ with integer $n$ (see solid red and blue curves), and these completely dominate the electronic peaks in the strong-coupling limit (solid blue). For smaller phonon bath temperatures, the resonances would be more pronounced for positive $\Delta \varepsilon$, since phonon emission into the bath is more likely (not shown). Phonon induced oscillations in the electronic current as a function of the level detuning have been seen in experiments with InAs and graphene double quantum dots. ${ }^{12}$ When we couple electronic transport to a continuum of phonon modes, these detailed oscillations cannot be resolved anymore (dashed curves in the background, see also the figure caption).

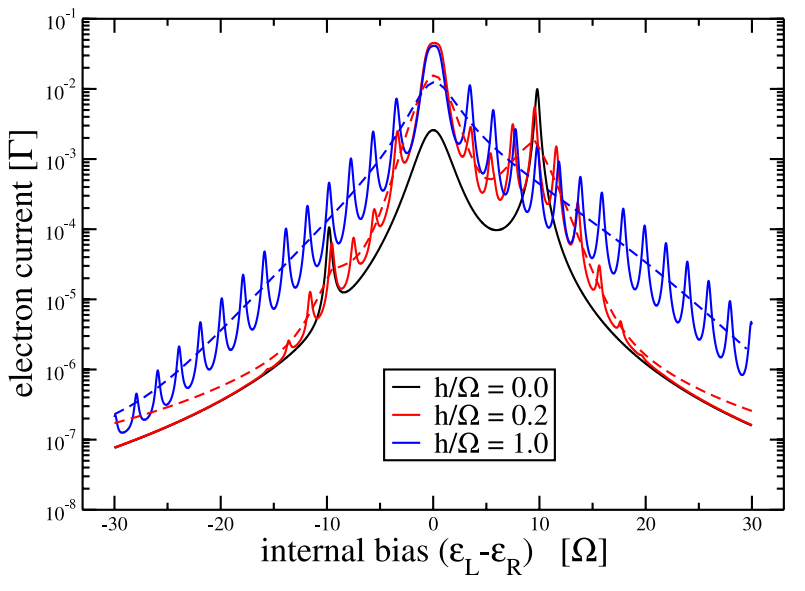

FIG. 2. Electron current in units of $\Gamma_{\mathrm{L}}=\Gamma_{\mathrm{R}}=\Gamma$ versus the internal bias $\Delta \varepsilon=\varepsilon_{\mathrm{L}}-\varepsilon_{\mathrm{R}}$ in units of $\Omega$. All graphs are evaluated far from the electronic wideband limit $\delta_{\mathrm{L}} / \Omega=\delta_{\mathrm{R}} / \Omega=\delta / \Omega=0.1$. The electron-phonon couplings, left and right, are chosen equal $h_{q \mathrm{~L}} / \Omega=h_{q \mathrm{R}} / \Omega=h / \Omega$. The black line shows pure electronic transport decoupled from the phonon bath, $h / \Omega=0$. Due to the sharp Lorentzian shaped electronic tunneling rates observe two prominent electronic resonances. When adding coupling to a single phonon mode (solid curves), we see that additional resonances appear. Caused by the on-site level configuration and large phonon bath temperatures $\left(\beta_{\mathrm{ph}} \Omega=0.1\right)$ the resonances approximately symmetric in $\Delta \varepsilon$. At strong electron-phonon coupling, resonances appear over the whole internal bias range (blue line). This is different for coupling to a continuum phonon reservoir (dashed curves in the background), where no additional resonances are found. Other parameters are chosen as, $\Gamma / \Omega=0.01, U / \Omega=5.0, T_{\mathrm{c}} / \Omega=1.0, \Phi=0, \beta_{\mathrm{el}}=\beta_{\mathrm{ph}}$ (implying $\Gamma \beta_{\mathrm{el}}=10^{-3}$ and $\left.\varepsilon_{+}-\varepsilon_{-} \geq 2\left|T_{\mathrm{c}}\right|\right)$. Continuum parameters have been adjusted such that $\int_{0}^{\infty} J_{\sigma}(\omega) d \omega=|h|^{2}$ and $\int_{0}^{\infty} J_{\sigma}(\omega) / \omega d \omega=|h|^{2} / \Omega$.

\section{B. Current/dephasing rate versus external bias}

Typically, the current as a function of the external bias can be used to obtain internal system parameters via transport spectroscopy: transition frequencies of the system entering the transport window will-at sufficiently small temperaturesinduce steps in the current. In Fig. 3 we display the electronic matter current for different electron-phonon coupling strengths. Whereas - as a consequence of the phonon presence-the single-mode version (solid curves) displays now many additional plateaus that allow, e.g., for spectroscopy of the phonon frequency, the continuous phonon versions (dashed and dotted) only display a suppression of the current for small bias. This phenomenon-termed Franck-Condon blockade $^{83}$-is also observed when the phonons are taken into account dynamically.

Computing the dynamics of the coherences $\left\langle-\left|\rho_{\mathrm{S}}(t)\right|+\right\rangle$ $=\left(\left\langle+\left|\rho_{\mathrm{S}}(t)\right|-\right\rangle\right)^{*}$ yields a simple time evolution $\dot{\boldsymbol{\rho}}_{-+}(t)$ $=-\gamma \rho_{-+}(t)$, see Eq. (26). This implies that the absolute square of $\rho_{-+}(t)$ decays exponentially with $\left|\rho_{-+}(t)\right|^{2}$ $=e^{-2 \mathfrak{R}(\gamma) t}\left|\boldsymbol{\rho}_{-+}(0)\right|^{2}$, where this dephasing is induced by both electronic and phononic reservoirs. The dephasing rate $2 \mathfrak{R}(\gamma)$ is a measure for the decay of the superposition of the states $|-\rangle$ and $|+\rangle$ to a classical mixture. When we neglect the asymmetry of the coupling $h_{q L}=h_{q R}$, the phonon correlation functions for the internal jumps vanish, and in consequence also the internal transition rates $\gamma_{-+,-+}$and $\gamma_{+-,+-}$in Eq. (26) vanish. Since, furthermore, diagonal matrix elements of the first four system coupling operators (such as, e.g., $\left\langle-\left|d_{L}\right|-\right\rangle$ ) vanish throughout, it also follows that $\gamma_{--,++}=0$, and we obtain for the dephasing rate 


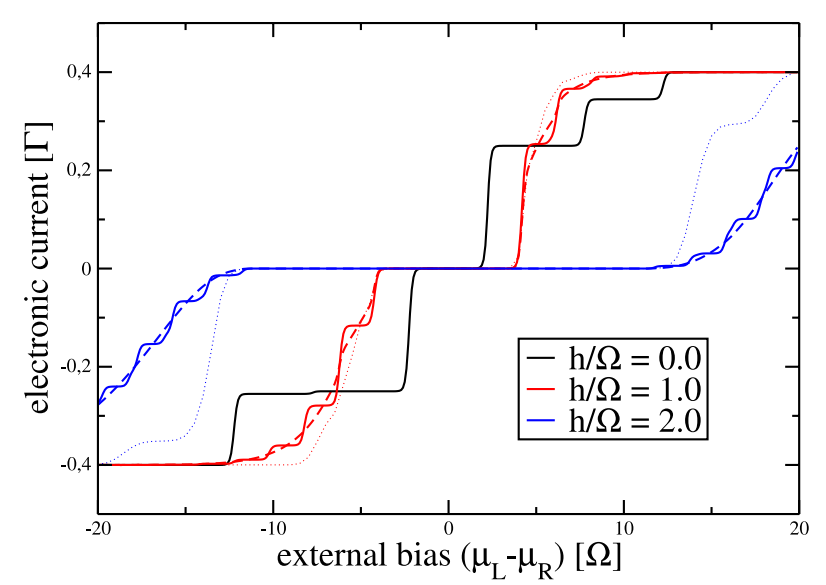

FIG. 3. Plot of the electronic current versus the external bias voltage for different electron-phonon coupling strengths. With increasing coupling strength, the steps corresponding to the bare electronic transitions (black curve) become supplemented by additional plateaus, accounting for an increasing number of phonons involved in the transport process. The width of these smaller steps allows to determine the phonon frequency. Consistently, the continuum phonon reservoir (dashed curves in background) does not exhibit these smaller steps. Other parameters are chosen as, $\Gamma / \Omega=0.01, T_{\mathrm{c}} / \Omega=1.0, \beta_{\mathrm{L}} \Omega=\beta_{\mathrm{R}} \Omega=\beta_{\mathrm{ph}} \Omega=20.0, \delta_{\mathrm{L}} / \Omega=\delta_{\mathrm{R}} / \Omega \rightarrow \infty$, $\varepsilon_{\mathrm{L}} / \Omega=-\varepsilon_{\mathrm{R}} / \Omega=0.5, U / \Omega=5.0, \Phi=0.0$ (implying $\Gamma \beta_{\mathrm{el}}=0.2$ and $\varepsilon_{+}-\varepsilon_{-}$ $\left.=\sqrt{5}\left|T_{\mathrm{c}}\right|\right)$. Continuum parameters were adjusted such that $\int_{0}^{\infty} J_{\sigma}(\omega) d \omega$ $=|h|^{2}$ and $\int_{0}^{\infty} J_{\sigma}(\omega) / \omega d \omega=|h|^{2} / \Omega$ (dashed curves). Further approximating the continuum phonon correlation function with a single Gaussian as described in Sec. III D yields for small bias quite analogous results (dotted curves).

$$
\mathcal{R}=\left[\Gamma_{\mathrm{L}}^{0-}+\Gamma_{\mathrm{R}}^{0-}+\Gamma_{\mathrm{L}}^{0+}+\Gamma_{\mathrm{R}}^{0+}+\Gamma_{\mathrm{L}}^{2-}+\Gamma_{\mathrm{R}}^{2-}+\Gamma_{\mathrm{L}}^{2+}+\Gamma_{\mathrm{R}}^{2+}\right]
$$

where we have used the abbreviations defined in Eq. (27). The phonon plateaus are also very well visible in the dephasing rate, see Fig. 4. Counter-intuitively, when we increase the electronphonon coupling, the dephasing rate first decreases before it increases again (compare orange curves in the inset). This suppression occurs in the current blockade regime. Interestingly, the dephasing rate becomes much smaller than the equilibrium dephasing rate observed without phonons. Thus, we find that while increasing the coupling strength to the phonon reservoir, the model effectively shows a decrease of the dephasing rate which is in stark contrast to general expectations. We attribute this behaviour to the conditioned state of the phonon reservoir. A more intuitive explanation is that the FranckCondon blockade prevents transport through the charge qubit and thereby also transport-associated decoherence.

\section{Thermoelectric generator}

Multi-terminal nanostructures may serve as nanomachines converting, e.g., temperature gradients into electric power. Here, we consider the case where a hot phonon bath and cold electronic reservoirs may induce an electronic current at vanishing bias-or even a current against a finite bias generating useful power. We note that whereas for a single-electron transistor (with its always-symmetric electron-phonon coupling) one would require non-flat electronic tunneling rates to see such an effect, this is different in the present model when we apply it to the case of a continuous phonon spectrum. Formally, we consider in Eq. (57) a situation where the matter current

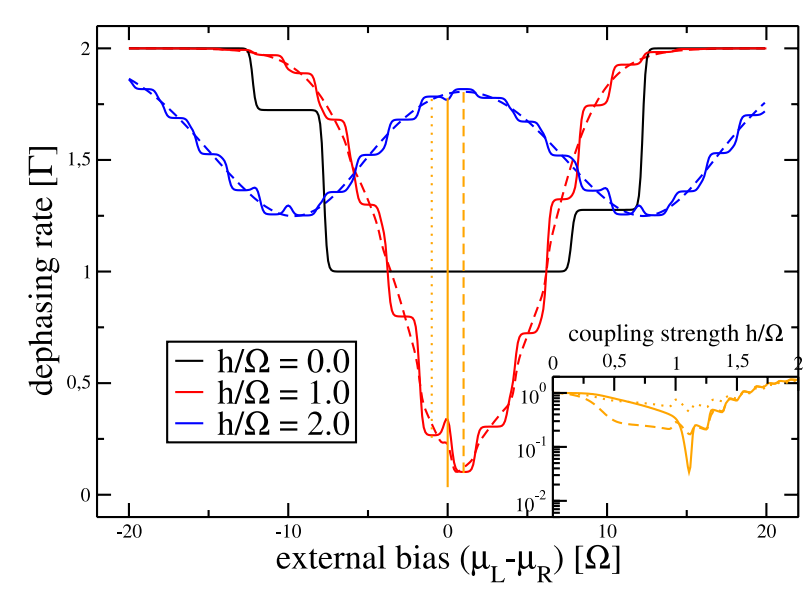

FIG. 4. Dephasing rate $\mathcal{R}$ in units of $\Gamma_{L}=\Gamma_{R}=\Gamma$ versus the external bias voltage $V$ in units of $\Omega$. The black reference curve shows the dephasing rate for pure electronic transport, $h_{q \mathrm{~L}}=h_{q \mathrm{R}}=h=0.0$. Without phonon coupling, the dephasing rate where transport is dominated by the transitions $|0\rangle \rightarrow|-\rangle,|+\rangle$ lies on the same level as the equilibrium dephasing rate, such that no step is visible. If we increase the electron-phonon coupling $h_{q \mathrm{~L}}=h_{q \mathrm{R}}$ we see a severe modulation of the curves. In the Franck-Condon regime around $V=0.0$ (vertical orange lines mark maximum and minimum dephasing rates in the interval $h / \Omega \in[0,2]$ ), the dephasing rate becomes suppressed for intermediate electron-phonon coupling strengths (see the inset for the dephasing rate at $V \in\{-\Omega, 0,+\Omega\})$. Other parameters are chosen as, $\Gamma / \Omega=0.01, T_{\mathrm{c}} / \Omega=1.0, \beta_{\mathrm{L}} \Omega=\beta_{\mathrm{R}} \Omega=\beta_{\mathrm{ph}} \Omega=20.0, \delta_{\mathrm{L}} / \Omega=\delta_{\mathrm{R}} / \Omega \rightarrow \infty$, $\varepsilon_{\mathrm{L}} / \Omega=-\varepsilon_{\mathrm{R}} / \Omega=0.5, U / \Omega=5.0, \Phi=0.0$ (implying $\Gamma \beta_{\mathrm{el}}=0.2$ and $\varepsilon_{+}-\varepsilon_{-}$ $\left.=\sqrt{5}\left|T_{\mathrm{c}}\right|\right)$. Continuum parameters were adjusted such that $\int_{0}^{\infty} J_{\sigma}(\omega) d \omega$ $=|h|^{2}$ and $\int_{0}^{\infty} J_{\sigma}(\omega) / \omega d \omega=|h|^{2} / \Omega$.

$I_{\mathrm{M}}=I_{\mathrm{M}}^{\mathrm{L}}$ from left to right is negative although $\mu_{\mathrm{L}}<\mu_{\mathrm{R}}$. This is for $\beta_{\mathrm{el}}<\beta_{\mathrm{ph}}$ only possible when heat flows out of the hot phonon reservoir, with use of Eq. (57) more precisely when $I_{\mathrm{E}}^{\mathrm{ph}} \geq-\frac{\beta_{\mathrm{el}}}{\beta_{\mathrm{el}}-\beta_{\mathrm{ph}}}\left(\mu_{L}-\mu_{R}\right) I_{\mathrm{M}}^{\mathrm{L}}>0$. To quantify the performance of such a device, it is instructive to relate the power output $P_{\text {out }}=-I_{\mathrm{M}}^{\mathrm{L}}\left(\mu_{\mathrm{L}}-\mu_{\mathrm{R}}\right)=-I_{\mathrm{M}} V$ to the heat entering from the hot phonon reservoir $Q=I_{\mathrm{E}}^{\mathrm{ph}}$. Positivity of entropy production (57) then grants that the efficiency of this process

$$
\eta=\frac{P_{\text {out }}}{Q}=-\frac{I_{\mathrm{M}} V}{I_{\mathrm{E}}^{\mathrm{ph}}} \leq 1-\frac{T_{\mathrm{el}}}{T_{\mathrm{ph}}}=\eta_{\mathrm{Ca}}
$$

is upper-bounded by Carnot efficiency. In general however, the efficiency can be significantly smaller, as is illustrated in Fig. 5. In fact, the inset shows that Carnot efficiency is not even reached at the new equilibrium, where the electronic matter current vanishes. Formally, this is due to the fact that-in contrast to previous weak-coupling models ${ }^{76,84}$ - the total entropy production does not vanish at this point. This is somewhat expected, since due to the presence of phonons, our model does not obey the tight-coupling condition. ${ }^{85}$

\section{SUMMARY}

We have investigated coherent electronic transport strongly coupled to vibrations. To obtain a thermodynamically consistent master equation, the secular-approximation has to be performed in the new system basis that arises after the polaron transform. The method presented here yields a low dimensional master equation in Lindblad form which accounts for thermodynamic consistency, although in the original frame the phonons are in a displaced thermal state. Thermodynamic 


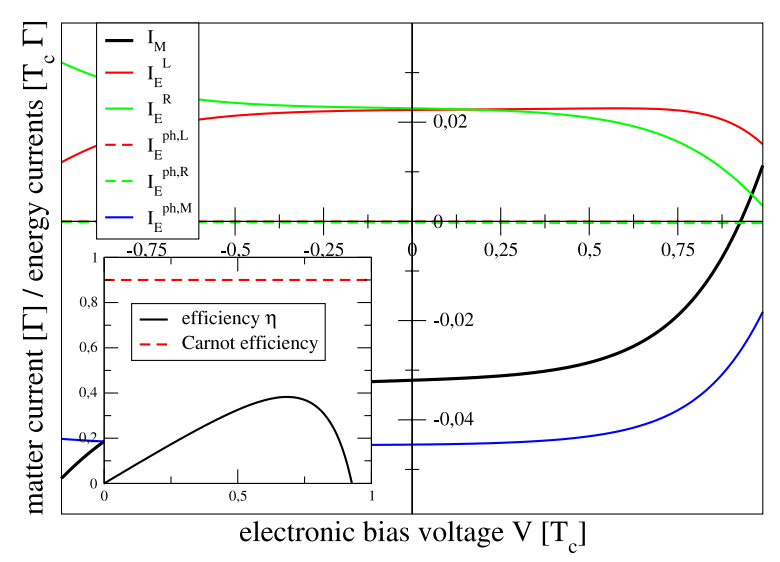

FIG. 5. Plot of the matter and energy currents for a hot phonon and cold electronic reservoirs versus electronic bias voltage. In the lower right quadrant, the electronic matter current (bold black) runs against a potential gradient, thereby generating power $P_{\text {out }}=-I_{\mathrm{M}} V$. The first law manifests in the fact that all energy currents add up to zero. Parameters have been chosen such that the internal phonon-assisted transitions between eigenstates $|-\rangle$ and $|+\rangle$ dominate the phonon heat flow (solid blue versus dashed curves for external jumps). Relating the power output with the heat input from the phonon reservoir $Q=+\left(I_{E}^{\mathrm{ph}, \mathrm{L}}+I_{E}^{\mathrm{ph}, \mathrm{R}}+I_{\mathrm{E}}^{\mathrm{ph}, \mathrm{M}}\right)$, we see that the efficiency of this process (inset, for positive bias voltage only) remains significantly below Carnot efficiency. Other parameters, $\Gamma_{\mathrm{L}}=\Gamma_{\mathrm{R}}=\Gamma=0.01 T_{\mathrm{c}}, J_{\mathrm{L}} T_{\mathrm{c}}^{2}=J_{\mathrm{R}} T_{\mathrm{c}}^{2}=0.001, J_{0} T_{\mathrm{c}}^{2}=1.0$, $w_{\mathrm{c}}^{\mathrm{L}}=w_{\mathrm{c}}^{\mathrm{R}}=w_{\mathrm{c}}=1.0 T_{\mathrm{c}}, \varepsilon_{\mathrm{L}}=+0.5 T_{\mathrm{c}}=-\varepsilon_{\mathrm{R}}, U=5.0 T_{\mathrm{c}}, \beta_{\mathrm{L}} T_{\mathrm{c}}=\beta_{\mathrm{R}} T_{\mathrm{c}}=10.0$, $\beta_{\mathrm{ph}} T_{\mathrm{c}}=1.0$ (implying $\beta_{\mathrm{el}} \Gamma=0.1$ and $\varepsilon_{+}-\varepsilon_{-}=\sqrt{5}\left|T_{\mathrm{c}}\right|$ ).

consistency has been demonstrated by an analytic proof of the fluctuation theorem for entropy production.

Using the full counting statistics, we computed the electronic current versus internal and external bias and reproduced electron-phonon-induced features such as oscillations versus the internal bias and signatures of Franck-Condon blockade. We stress that the description of this rich dynamics required only the four states of the double quantum dot to be taken into account dynamically.

We have found that the dephasing rate of coherences in the pointer basis behaves in some regimes counter-intuitively as a function of the electron-phonon coupling strength. A simple intuitive explanation for this behaviour is that the FranckCondon blockade stabilizes coherences, thereby also blocking transport through the DQD circuit.

The analysis of the entropy production in the polaron master equation has allowed us to study the performance of the system when viewed as a thermoelectric generator converting a temperature gradient into electric power. We have found that in the strong-coupling regime, the system deviates strongly from tight-coupling between energy and matter current, and consequently, the efficiency for this process was found to be significantly below Carnot efficiency.

Finally, we want to mention that our method can be generalized to more complex systems and thus allows applications in a variety of transport setups involving phonons such as molecules.

\section{ACKNOWLEDGMENTS}

T.B. and G.S. gratefully acknowledge financial support by the DFG (SFB 910, GRK 1588, SCHA 1646/3-1). M.E. has been supported by the National Research Fund, Luxembourg, in the frame of Project No. FNR/A11/02.

\section{APPENDIX A: POLARON TRANSFORMATION}

We consider the polaron transformation

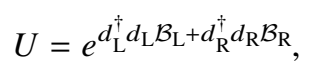

with the fermionic annihilation operators $d_{\sigma}$ and the bosonic operators

$$
\mathcal{B}_{\sigma}=\sum_{q}\left(\frac{h_{q, \sigma}^{*}}{\omega_{q}} a_{q}^{\dagger}-\frac{h_{q, \sigma}}{\omega_{q}} a_{q}\right)
$$

with bosonic annihilation operators $a_{q}$. To calculate the transformation rules, we recall the $\mathrm{BCH}$ relation

$$
e^{X} Y e^{-X}=\sum_{n=0}^{\infty} \frac{1}{n !}[X, Y]_{n},
$$

with the short-hand notation $[X, Y]_{n+1}=\left[X,[X, Y]_{n}\right]$ and $[X, Y]_{0}=Y$. We first note that the exponential in the polaron transformation can be written in a separated fashion

$$
\begin{aligned}
U & =e^{d_{\mathrm{L}}^{\dagger} d_{\mathrm{L}} \mathcal{B}_{\mathrm{L}}} e^{d_{\mathrm{R}}^{\dagger} d_{\mathrm{R}} \mathcal{B}_{\mathrm{R}}} e^{-d_{\mathrm{L}}^{\dagger} d_{\mathrm{L}} d_{\mathrm{R}}^{\dagger} d_{\mathrm{R}}\left[\mathcal{B}_{\mathrm{L}}, \mathcal{B}_{\mathrm{R}}\right] / 2} \\
& \equiv U_{\mathrm{L}} U_{\mathrm{R}} U_{\mathrm{LR}}, \\
U_{\mathrm{LR}} & =e^{d_{\mathrm{L}}^{\dagger} d_{\mathrm{L}} d_{\mathrm{R}}^{\dagger} d_{\mathrm{R}} \mathrm{i} \Phi}, \\
\mathrm{i} \Phi & \equiv\left[\mathcal{B}_{\mathrm{R}}, \mathcal{B}_{\mathrm{L}}\right] / 2,
\end{aligned}
$$

where it is easy to show that $\Phi^{*}=\Phi$. Consequently, the adjoint operator is given by

$$
U^{\dagger}=U_{\mathrm{LR}}^{\dagger} U_{\mathrm{R}}^{\dagger} U_{\mathrm{L}}^{\dagger},
$$

and we note that $\left[U_{\mathrm{LR}}, U_{\mathrm{L}}\right]=\left[U_{\mathrm{LR}}, U_{\mathrm{R}}\right]=0$. Alternatively, we can also split the unitary transformation according to

$$
U=U_{\mathrm{R}} U_{\mathrm{L}} U_{\mathrm{LR}}^{\dagger}, \quad U^{\dagger}=U_{\mathrm{LR}} U_{\mathrm{L}}^{\dagger} U_{\mathrm{R}}^{\dagger},
$$

where again $\left[U_{\mathrm{LR}}, U_{\mathrm{L}}^{\dagger}\right]=\left[U_{\mathrm{LR}}, U_{\mathrm{R}}^{\dagger}\right]=0$ holds.

\section{Left mode operators}

We consider the action of the Polaron transformation on the left fermionic annihilation operator

$$
\begin{aligned}
U d_{\mathrm{L}} U^{\dagger} & =U_{\mathrm{L}} U_{\mathrm{R}} U_{\mathrm{LR}} d_{\mathrm{L}} U_{\mathrm{LR}}^{\dagger} U_{\mathrm{R}}^{\dagger} U_{\mathrm{L}}^{\dagger}=U_{\mathrm{L}} U_{\mathrm{LR}} d_{\mathrm{L}} U_{\mathrm{LR}}^{\dagger} U_{\mathrm{L}}^{\dagger} \\
& =U_{\mathrm{L}} d_{\mathrm{L}} e^{-d_{\mathrm{R}}^{\dagger} d_{\mathrm{R}} \mathrm{i} \Phi} U_{\mathrm{L}}^{\dagger}=U_{\mathrm{L}} d_{\mathrm{L}} U_{\mathrm{L}}^{\dagger} e^{-d_{\mathrm{R}}^{\dagger} d_{\mathrm{R}} \mathrm{i} \Phi} \\
& =d_{\mathrm{L}} e^{-\mathcal{B}_{\mathrm{L}}} e^{-d_{\mathrm{R}}^{\dagger} d_{\mathrm{R}} \mathrm{i} \Phi} .
\end{aligned}
$$

The left fermionic creation operator then transforms according to

$$
U d_{\mathrm{L}}^{\dagger} U^{\dagger}=d_{\mathrm{L}}^{\dagger} e^{+\mathcal{B}_{\mathrm{L}}} e^{+d_{\mathrm{R}}^{\dagger} d_{\mathrm{R}}^{\mathrm{i} \Phi}}
$$

\section{Right mode operators}

In a similar fashion, we evaluate the transformation of the right fermionic annihilation operator

$$
\begin{aligned}
U d_{\mathrm{R}} U^{\dagger} & =U_{\mathrm{R}} U_{\mathrm{L}} U_{\mathrm{LR}}^{\dagger} d_{\mathrm{R}} U_{\mathrm{LR}} U_{\mathrm{L}}^{\dagger} U_{\mathrm{R}}^{\dagger}=U_{\mathrm{R}} U_{\mathrm{LR}}^{\dagger} d_{\mathrm{R}} U_{\mathrm{LR}} U_{\mathrm{R}}^{\dagger} \\
& =U_{\mathrm{R}} d_{\mathrm{R}} e^{+d_{\mathrm{L}}^{\dagger} d_{\mathrm{L}} \mathrm{i} \Phi} U_{\mathrm{R}}^{\dagger}=U_{\mathrm{R}} d_{\mathrm{R}} U_{\mathrm{R}}^{\dagger} e^{+d_{\mathrm{L}}^{\dagger} d_{\mathrm{L}} \mathrm{i} \Phi} \\
& =d_{\mathrm{R}} e^{-\mathcal{B}_{\mathrm{R}}} e^{+d_{\mathrm{L}}^{\dagger} d_{\mathrm{L}} \mathrm{i} \Phi}
\end{aligned}
$$

and the adjoint operator becomes 


$$
U d_{\mathrm{R}}^{\dagger} U^{\dagger}=d_{\mathrm{R}}^{\dagger} e^{+\mathcal{B}_{\mathrm{R}}} e^{-d_{\mathrm{L}}^{\dagger} d_{\mathrm{L}} \mathrm{i} \Phi}
$$

\section{Bosonic operators}

For the bosonic annihilation operator, we obtain

$$
\begin{aligned}
U a_{q} U^{\dagger} & =U_{\mathrm{L}} U_{\mathrm{R}} U_{\mathrm{LR}} a_{q} U_{\mathrm{LR}}^{\dagger} U_{\mathrm{R}}^{\dagger} U_{\mathrm{L}}^{\dagger}=U_{\mathrm{L}} U_{\mathrm{R}} d_{\mathrm{L}} U_{\mathrm{R}}^{\dagger} U_{\mathrm{L}}^{\dagger} \\
& =U_{\mathrm{L}}\left[a_{q}-\frac{h_{q, \mathrm{R}}^{*}}{\omega_{q}} d_{\mathrm{R}}^{\dagger} d_{\mathrm{R}}\right] U_{\mathrm{L}}^{\dagger} \\
& =U_{\mathrm{L}} a_{q} U_{\mathrm{L}}^{\dagger}-\frac{h_{q, \mathrm{R}}^{*}}{\omega_{q}} d_{\mathrm{R}}^{\dagger} d_{\mathrm{R}} \\
& =a_{q}-\frac{h_{q, \mathrm{~L}}^{*}}{\omega_{q}} d_{\mathrm{L}}^{\dagger} d_{\mathrm{L}}-\frac{h_{q, \mathrm{R}}^{*}}{\omega_{q}} d_{\mathrm{R}}^{\dagger} d_{\mathrm{R}}
\end{aligned}
$$

and similarly for the creation operator

$$
U a_{q}^{\dagger} U^{\dagger}=a_{q}^{\dagger}-\frac{h_{q, \mathrm{~L}}}{\omega_{q}} d_{\mathrm{L}}^{\dagger} d_{\mathrm{L}}-\frac{h_{q, \mathrm{R}}}{\omega_{q}} d_{\mathrm{R}}^{\dagger} d_{\mathrm{R}} .
$$

\section{Polaron transformation of the DQD Hamiltonian}

The total Hamiltonian of the DQD is given by

$$
\begin{aligned}
\mathcal{H}= & \sum_{k \sigma} \varepsilon_{k \sigma} c_{k \sigma}^{\dagger} c_{k \sigma}+\sum_{q} \omega_{q} a_{q}^{\dagger} a_{q} \\
& +\varepsilon_{\mathrm{L}} d_{\mathrm{L}}^{\dagger} d_{\mathrm{L}}+\varepsilon_{\mathrm{R}} d_{\mathrm{R}}^{\dagger} d_{\mathrm{R}}+U d_{\mathrm{L}}^{\dagger} d_{\mathrm{L}} d_{\mathrm{R}}^{\dagger} d_{\mathrm{R}} \\
& +T_{\mathrm{c}}\left(d_{\mathrm{L}} d_{\mathrm{R}}^{\dagger}+d_{\mathrm{R}} d_{\mathrm{L}}^{\dagger}\right)+\sum_{k \sigma}\left(t_{k \sigma} d_{\sigma} c_{k \sigma}^{\dagger}+\text { h.c. }\right) \\
& +\sum_{q \sigma}\left(h_{q \sigma} a_{q}+h_{q \sigma}^{*} a_{q}^{\dagger}\right) d_{\sigma}^{\dagger} d_{\sigma} .
\end{aligned}
$$

Applying the polaron transformation to the total Hamiltonian $\overline{\mathcal{H}}=U \mathcal{H} U^{\dagger}$ implies that some parts of the Hamiltonian will change. In particular, we have for the free bosonic Hamiltonian

$$
\begin{aligned}
H_{\mathrm{ph}}^{\prime}= & \sum_{q} \omega_{q}\left(a_{q}^{\dagger}-\frac{h_{q \mathrm{~L}}}{\omega_{q}} d_{\mathrm{L}}^{\dagger} d_{\mathrm{L}}-\frac{h_{q \mathrm{R}}}{\omega_{q}} d_{\mathrm{R}}^{\dagger} d_{\mathrm{R}}\right) \\
& \times\left(a_{q}-\frac{h_{q \mathrm{~L}}^{*}}{\omega_{q}} d_{\mathrm{L}}^{\dagger} d_{\mathrm{L}}-\frac{h_{q \mathrm{R}}^{*}}{\omega_{q}} d_{\mathrm{R}}^{\dagger} d_{\mathrm{R}}\right),
\end{aligned}
$$

for the electronic inter-dot tunneling Hamiltonian

$$
\begin{aligned}
H_{T}^{\prime}= & T_{\mathrm{c}} d_{\mathrm{L}} e^{-\left(d_{\mathrm{L}}^{\dagger} d_{\mathrm{L}}+d_{\mathrm{R}}^{\dagger} d_{\mathrm{R}}\right) \mathrm{i} \Phi} d_{\mathrm{R}}^{\dagger} e^{-\mathcal{B}_{\mathrm{L}}} e^{+\mathcal{B}_{\mathrm{R}}} \\
& +T_{\mathrm{c}} d_{\mathrm{R}} e^{+\left(d_{\mathrm{L}}^{\dagger} d_{\mathrm{L}}+d_{\mathrm{R}}^{\dagger} d_{\mathrm{R}}\right) \mathrm{i} \Phi} d_{\mathrm{L}}^{\dagger} e^{-\mathcal{B}_{\mathrm{R}}} e^{+\mathcal{B}_{\mathrm{L}}} \\
= & T_{\mathrm{c}} e^{-2 \mathrm{i} \Phi} d_{\mathrm{L}} d_{\mathrm{R}}^{\dagger} e^{-\mathcal{B}_{\mathrm{L}}} e^{+\mathcal{B}_{\mathrm{R}}} \\
& +T_{\mathrm{c}} e^{+2 \mathrm{i} \Phi} d_{\mathrm{R}} d_{\mathrm{L}}^{\dagger} e^{-\mathcal{B}_{\mathrm{R}}} e^{+\mathcal{B}_{\mathrm{L}}},
\end{aligned}
$$

for the electron-lead tunneling Hamiltonian

$$
\begin{aligned}
H_{V}^{\prime}= & \sum_{k}\left(t_{k \mathrm{~L}} d_{\mathrm{L}} e^{\left.-d_{\mathrm{R}}^{\dagger} d_{\mathrm{R}}^{\mathrm{i} \Phi} e^{-\mathcal{B}_{\mathrm{L}}} c_{k L}^{\dagger}+\text { h.c. }\right)}\right. \\
& +\sum_{k}\left(t_{k \mathrm{R}} d_{\mathrm{R}} e^{+d_{\mathrm{L}}^{\dagger} d_{\mathrm{L}} \mathrm{i} \Phi} e^{-\mathcal{B}_{\mathrm{R}}} c_{k R}^{\dagger}+\text { h.c. }\right)
\end{aligned}
$$

and for the electron-phonon interaction

$$
\begin{aligned}
H_{\mathrm{e}-\mathrm{ph}}^{\prime}= & \sum_{q \sigma}\left(h_{q \sigma} a_{q}+h_{q \sigma}^{*} a_{q}^{\dagger}\right) d_{\sigma}^{\dagger} d_{\sigma} \\
& -\sum_{q \sigma} h_{q \sigma}\left(\frac{h_{q \mathrm{~L}}^{*}}{\omega_{q}} d_{\mathrm{L}}^{\dagger} d_{\mathrm{L}}+\frac{h_{q \mathrm{R}}^{*}}{\omega_{q}} d_{\mathrm{R}}^{\dagger} d_{\mathrm{R}}\right) d_{\sigma}^{\dagger} d_{\sigma} \\
& -\sum_{q \sigma} h_{q \sigma}^{*}\left(\frac{h_{q \mathrm{~L}}}{\omega_{q}} d_{\mathrm{L}}^{\dagger} d_{\mathrm{L}}+\frac{h_{q \mathrm{R}}}{\omega_{q}} d_{\mathrm{R}}^{\dagger} d_{\mathrm{R}}\right) d_{\sigma}^{\dagger} d_{\sigma}
\end{aligned}
$$

For the sum of the free phonon and the electron-phonon interaction Hamiltonians, we obtain

$$
\begin{aligned}
H_{\mathrm{ph}}^{\prime}+H_{\mathrm{e}-\mathrm{ph}}^{\prime}= & \sum_{q} \omega_{q} a_{q}^{\dagger} a_{q} \\
& -\sum_{q}\left(\frac{\left|h_{q \mathrm{~L}}\right|^{2}}{\omega_{q}} d_{\mathrm{L}}^{\dagger} d_{\mathrm{L}}+\frac{\left|h_{q \mathrm{R}}\right|^{2}}{\omega_{q}} d_{\mathrm{R}}^{\dagger} d_{\mathrm{R}}\right) \\
& -\sum_{q} \frac{h_{q \mathrm{~L}}^{*} h_{q \mathrm{R}}+h_{q \mathrm{~L}} h_{q \mathrm{R}}^{*}}{\omega_{q}} d_{\mathrm{L}}^{\dagger} d_{\mathrm{L}} d_{\mathrm{R}}^{\dagger} d_{\mathrm{R}} .
\end{aligned}
$$

Therefore, the total Hamiltonian after the polaron transformation reads

$$
\begin{aligned}
H= & \sum_{k \sigma} \varepsilon_{k \sigma} c_{k \sigma}^{\dagger} c_{k \sigma}+\sum_{q} \omega_{q} a_{q}^{\dagger} a_{q}+\bar{\varepsilon}_{\mathrm{L}} d_{\mathrm{L}}^{\dagger} d_{\mathrm{L}}+\bar{\varepsilon}_{\mathrm{R}} d_{\mathrm{R}}^{\dagger} d_{\mathrm{R}}+\bar{U} d_{\mathrm{L}}^{\dagger} d_{\mathrm{L}} d_{\mathrm{R}}^{\dagger} d_{\mathrm{R}}+T_{\mathrm{c}} e^{-2 \mathrm{i} \Phi} d_{\mathrm{L}} d_{\mathrm{R}}^{\dagger} e^{-\mathcal{B}_{\mathrm{L}}} e^{+\mathcal{B}_{\mathrm{R}}} \\
& +T_{\mathrm{c}} e^{+2 \mathrm{i} \Phi} d_{\mathrm{R}} d_{\mathrm{L}}^{\dagger} e^{-\mathcal{B}_{\mathrm{R}}} e^{+\mathcal{B}_{\mathrm{L}}}+\sum_{k}\left(t_{k \mathrm{~L}} d_{\mathrm{L}} e^{-d_{\mathrm{R}}^{\dagger} d_{\mathrm{R}}{ }^{\mathrm{i} \Phi}} e^{-\mathcal{B}_{\mathrm{L}}} c_{k L}^{\dagger}+\text { h.c. }\right)+\sum_{k}\left(t_{k \mathrm{R}} d_{\mathrm{R}} e^{+d_{\mathrm{L}}^{\dagger} d_{\mathrm{L}} \mathrm{i} \Phi} e^{-\mathcal{B}_{\mathrm{R}}} c_{k R}^{\dagger}+\text { h.c. }\right)
\end{aligned}
$$

with renormalized on-site energies (10) and the Coulomb interaction (11). When furthermore one demands that all expectation values of reservoir coupling operators should vanish (see below), one arrives at the splitting into system, reservoir, and interaction parts used in the paper.

\section{APPENDIX B: SHIFT FACTOR}

We use that for a thermal state $\rho \propto e^{-\beta \sum_{q} \omega_{q} a_{q}^{\dagger} a_{q}}$, one has for all complex-valued numbers $\alpha_{q}$,

$$
\left\langle e^{-\sum_{q}\left(\alpha_{q} a_{q}^{\dagger}-\alpha_{q}^{*} a_{q}\right)}\right\rangle=e^{-\Sigma_{q}\left|\alpha_{q}\right|^{2}\left[n_{\mathrm{B}}\left(\omega_{q}\right)+1 / 2\right]}
$$


with the Bose-distribution $n_{\mathrm{B}}\left(\omega_{q}\right)=\left[e^{\beta \omega_{q}}-1\right]^{-1}$. Applying that to the shift factor $\kappa$, for generality in the interaction picture, we obtain

$$
\begin{aligned}
& \kappa=\left\langle e^{-\mathcal{B}_{\mathrm{L}}(\tau)} e^{+\mathcal{B}_{\mathrm{R}}(\tau)}\right\rangle=\left\langle e^{-\mathcal{B}_{\mathrm{L}}(\tau)+\mathcal{B}_{\mathrm{R}}(\tau)}\right\rangle e^{\mathrm{i} \Phi}
\end{aligned}
$$

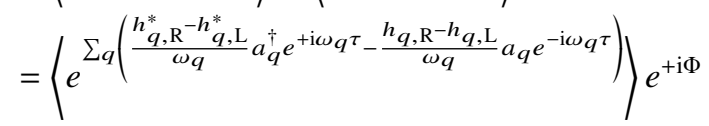

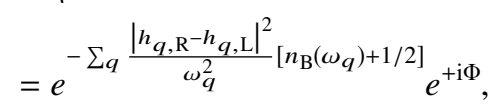

and see that $\kappa$ is independent of $\tau$. Therefore, we can already in the Schrödinger picture write the Hamiltonian in a way that is suitable for the derivation of a master equation with splitting into system, reservoir, and interaction parts given by Eqs. (9), (8), and (15) and (16) in the paper, respectively.

\section{APPENDIX C: INVERSE POLARON TRANSFORM}

To apply the inverse polaron transformation, it is useful to write it conditioned on the electronic occupation

$$
\begin{aligned}
U= & \mathbf{1}+d_{\mathrm{L}}^{\dagger} d_{\mathrm{L}}\left(e^{\mathcal{B}_{\mathrm{L}}}-\mathbf{1}\right)+d_{\mathrm{R}}^{\dagger} d_{\mathrm{R}}\left(e^{\mathcal{B}_{\mathrm{R}}}-\mathbf{1}\right) \\
& +d_{\mathrm{L}}^{\dagger} d_{\mathrm{L}} d_{\mathrm{R}}^{\dagger} d_{\mathrm{R}}\left(e^{\mathcal{B}_{\mathrm{L}}+\mathcal{B}_{\mathrm{R}}}-e^{\mathcal{B}_{\mathrm{L}}}-e^{\mathcal{B}_{\mathrm{R}}}+\mathbf{1}\right) \\
= & P_{0} \mathbf{1}+P_{\mathrm{L}} e^{\mathcal{B}_{\mathrm{L}}}+P_{\mathrm{R}} e^{\mathcal{B}_{\mathrm{R}}}+P_{2} e^{\mathcal{B}_{\mathrm{L}}+\mathcal{B}_{\mathrm{R}}},
\end{aligned}
$$

where with the projectors $P_{0}=\left(\mathbf{1}-d_{\mathrm{L}}^{\dagger} d_{\mathrm{L}}\right)\left(\mathbf{1}-d_{\mathrm{R}}^{\dagger} d_{\mathrm{R}}\right), P_{2}=d_{\mathrm{L}}^{\dagger}$ $d_{\mathrm{L}} d_{\mathrm{R}}^{\dagger} d_{\mathrm{R}}, P_{\mathrm{L}}=d_{\mathrm{L}}^{\dagger} d_{\mathrm{L}}\left(\mathbf{1}-d_{\mathrm{R}}^{\dagger} d_{\mathrm{R}}\right)$, and $P_{\mathrm{R}}=\left(\mathbf{1}-d_{\mathrm{L}}^{\dagger} d_{\mathrm{L}}\right) d_{\mathrm{R}}^{\dagger} d_{\mathrm{R}}$, it becomes visible that-depending on the system state in the localized basis - different unitary operations are applied on the reservoir. For the phonon reservoir state this implies

$$
\begin{aligned}
U^{\dagger} \rho_{\mathrm{B}}^{\mathrm{ph}} U= & P_{0} \otimes \rho_{\mathrm{B}}^{\mathrm{ph}}+P_{2} \otimes e^{-\left(\mathcal{B}_{\mathrm{L}}+\mathcal{B}_{\mathrm{R}}\right)} \rho_{\mathrm{B}}^{\mathrm{ph}} e^{+\left(\mathcal{B}_{\mathrm{L}}+\mathcal{B}_{\mathrm{R}}\right)} \\
& +P_{\mathrm{L}} \otimes e^{-\mathcal{B}_{\mathrm{L}}} \rho_{\mathrm{B}}^{\mathrm{ph}} e^{+\mathcal{B}_{\mathrm{L}}}+P_{\mathrm{R}} \otimes e^{-\mathcal{B}_{\mathrm{R}}} \rho_{\mathrm{B}}^{\mathrm{ph}} e^{+\mathcal{B}_{\mathrm{R}}}
\end{aligned}
$$

Considering that these unitary operations displace the phonons

$$
\begin{aligned}
e^{-\mathcal{B}_{\sigma}} a_{q}^{\dagger} a_{q} e^{+\mathcal{B}_{\sigma}} & =\left(a_{q}^{\dagger}+\frac{h_{q \sigma}}{\omega_{q}}\right)\left(a_{q}+\frac{h_{q \sigma}^{*}}{\omega_{q}}\right), \\
e^{-\mathcal{B}_{\mathrm{L}}-\mathcal{B}_{\mathrm{R}}} a_{q}^{\dagger} a_{q} e^{+\mathcal{B}_{\mathrm{L}}+\mathcal{B}_{\mathrm{R}}}= & \left(a_{q}^{\dagger}+\frac{h_{q \mathrm{~L}}+h_{q \mathrm{R}}}{\omega_{q}}\right) \\
& \times\left(a_{q}+\frac{h_{q \mathrm{~L}}^{*}+h_{q \mathrm{R}}^{*}}{\omega_{q}}\right),
\end{aligned}
$$

the reservoir state becomes the displaced thermal stateconditioned on the electronic occupation of the system.

Specifically, when in the localized basis, the system density matrix is written as

$$
\begin{aligned}
\rho_{\mathrm{S}}= & \rho_{0} P_{0}+\rho_{2} P_{2}+\rho_{\mathrm{L}} P_{\mathrm{L}}+\rho_{\mathrm{R}} P_{\mathrm{R}} \\
& +\rho_{\mathrm{LR}} P_{\mathrm{LR}}+\rho_{\mathrm{RL}} P_{\mathrm{RL}}
\end{aligned}
$$

with $P_{\mathrm{LR}}=|\mathrm{L}\rangle\langle\mathrm{R}|$ and $P_{\mathrm{RL}}=|\mathrm{R}\rangle\langle\mathrm{L}|$, it transforms according to

$$
\begin{aligned}
U^{\dagger} \rho_{\mathrm{S}} U= & \rho_{0} P_{0}+\rho_{2} P_{2}+\rho_{\mathrm{L}} P_{\mathrm{L}}+\rho_{\mathrm{R}} P_{\mathrm{R}}+\rho_{\mathrm{LR}} P_{\mathrm{L}} P_{\mathrm{LR}} P_{\mathrm{R}} \\
& \times e^{-\mathcal{B}_{\mathrm{L}}} e^{+\mathcal{B}_{\mathrm{R}}}+\rho_{\mathrm{RL}} P_{\mathrm{R}} P_{\mathrm{RL}} P_{\mathrm{L}} e^{-\mathcal{B}_{\mathrm{R}}} e^{+\mathcal{B}_{\mathrm{L}}} .
\end{aligned}
$$

This implies that the total system-phonon density matrix in the original frame is given by

$$
\begin{aligned}
\tilde{\rho}= & U^{\dagger} \rho_{\mathrm{S}} \otimes \mathbf{1} U U^{\dagger} \mathbf{1} \otimes \rho_{\mathrm{B}}^{\mathrm{ph}} U \\
= & \rho_{0} P_{0} \otimes \rho_{\mathrm{B}}^{\mathrm{ph}}+\rho_{2} P_{2} \otimes e^{-\left(\mathcal{B}_{\mathrm{L}}+\mathcal{B}_{\mathrm{R}}\right)} \rho_{\mathrm{B}}^{\mathrm{ph}} e^{+\left(\mathcal{B}_{\mathrm{L}}+\mathcal{B}_{\mathrm{R}}\right)} \\
& +\rho_{\mathrm{L}} P_{\mathrm{L}} \otimes e^{-\mathcal{B}_{\mathrm{L}}} \rho_{\mathrm{B}}^{\mathrm{ph}} e^{+\mathcal{B}_{\mathrm{L}}}+\rho_{\mathrm{R}} P_{\mathrm{R}} \otimes e^{-\mathcal{B}_{\mathrm{R}}} \rho_{\mathrm{B}}^{\mathrm{ph}} e^{+\mathcal{B}_{\mathrm{R}}} \\
& +\rho_{\mathrm{LR}} P_{\mathrm{LR}} \otimes e^{-\mathcal{B}_{\mathrm{L}}} \rho_{\mathrm{B}}^{\mathrm{ph}} e^{+\mathcal{B}_{\mathrm{R}}}+\rho_{\mathrm{RL}} P_{\mathrm{RL}} \otimes e^{-\mathcal{B}_{\mathrm{R}}} \rho_{\mathrm{B}}^{\mathrm{ph}} e^{+\mathcal{B}_{\mathrm{L}}} .
\end{aligned}
$$

\section{APPENDIX D: BATH CORRELATION FUNCTIONS}

\section{Phonon BCF}

We compute the expectation value of the phononic contribution in the lead-phonon bath correlation functions, cf. Sec. III B, given by

$$
\begin{aligned}
\mathcal{C}_{\mathrm{ph}}^{\sigma}= & \left\langle e^{-\mathcal{B}_{\sigma}(\tau)} e^{+\mathcal{B}_{\sigma}}\right\rangle=\left\langle e^{-\mathcal{B}_{\sigma}(\tau)+\mathcal{B}_{\sigma}}\right\rangle e^{-\left[\mathcal{B}_{\sigma}(\tau), \mathcal{B}_{\sigma}\right] / 2}=\left\langle e^{\sum_{q}\left[\frac{h_{q, \sigma}^{*}}{\omega_{q}}\left(1-e^{+\mathrm{i} \omega_{q} \tau}\right) a_{q}^{\dagger}-\frac{h_{q, \sigma}}{\omega_{q}}\left(1-e^{-\mathrm{i} \omega_{q} \tau}\right) a_{q}\right]}\right\rangle \\
& \times e^{-\mathrm{i} \sum_{q} \frac{\left|h_{q, \sigma}\right|^{2}}{\omega_{q}^{2}} \sin \left(\omega_{q} \tau\right)}=e^{-\sum_{q}\left|\frac{h_{q, \sigma}}{\omega_{q}}\left(1-e^{-\mathrm{i} \omega_{q} \tau}\right)\right|^{2}\left[n_{\mathrm{B}}\left(\omega_{q}\right)+1 / 2\right]} e^{-\mathrm{i} \sum_{q} \frac{\left|h_{q, \sigma}\right|^{2}}{\omega_{q}^{2}} \sin \left(\omega_{q} \tau\right)} \\
= & e^{-\sum_{q} \frac{\left|h_{q \sigma}\right|^{2}}{\omega_{q}^{2}}\left[2 n_{\mathrm{B}}\left(\omega_{q}\right)+1\right]} e^{\sum_{q} \frac{\left|h_{q \sigma}\right|^{2}}{\omega_{q}^{2}}\left\{n_{\mathrm{B}}\left(\omega_{q}\right) e^{+\mathrm{i} \omega_{q} \tau}+\left[n_{\mathrm{B}}\left(\omega_{q}\right)+1\right] e^{-\mathrm{i} \omega_{q} \tau}\right\}}
\end{aligned}
$$

And noting that it is invariant under the transformation $h_{q, \sigma} \rightarrow-h_{q, \sigma}$, we conclude

$$
\begin{aligned}
& \mathcal{C}_{12}(\tau)=\mathcal{C}_{\mathrm{ph}}^{\mathrm{L}}(\tau) \sum_{k}\left|t_{k, \mathrm{~L}}\right|^{2} f_{\mathrm{L}}\left(\varepsilon_{k, \mathrm{~L}}\right) e^{+\mathrm{i} \varepsilon_{k, \mathrm{~L}} \tau}, \mathcal{C}_{21}(\tau)=\mathcal{C}_{\mathrm{ph}}^{\mathrm{L}}(\tau) \sum_{k}\left|t_{k, \mathrm{~L}}\right|^{2}\left[1-f_{\mathrm{L}}\left(\varepsilon_{k, \mathrm{~L}}\right)\right] e^{-\mathrm{i} \varepsilon_{k, \mathrm{~L}} \tau} \\
& \mathcal{C}_{34}(\tau)=\mathcal{C}_{\mathrm{ph}}^{\mathrm{R}}(\tau) \sum_{k}\left|t_{k, \mathrm{R}}\right|^{2} f_{\mathrm{R}}\left(\varepsilon_{k, \mathrm{R}}\right) e^{+\mathrm{i} \varepsilon_{k, \mathrm{R}} \tau}, \mathcal{C}_{43}(\tau)=\mathcal{C}_{\mathrm{ph}}^{\mathrm{R}}(\tau) \sum_{k}\left|t_{k, \mathrm{R}}\right|^{2}\left[1-f_{\mathrm{R}}\left(\varepsilon_{k, \mathrm{R}}\right)\right] e^{-\mathrm{i} \varepsilon_{k, \mathrm{R}} \tau}
\end{aligned}
$$




\section{Inter-dot BCF}

We show explicitly that $\mathcal{C}_{55}(\tau)$ is given by Eq. (46),

$$
\begin{aligned}
\mathcal{C}_{55}(\tau)= & \left\langle e^{\mathcal{B}_{\mathrm{R}}(\tau)-\mathcal{B}_{\mathrm{L}}(\tau)+\mathcal{B}_{\mathrm{R}}-\mathcal{B}_{\mathrm{L}}}\right\rangle e^{+2 \mathrm{i} \Phi} e^{+\left[\mathcal{B}_{\mathrm{R}}(\tau)-\mathcal{B}_{\mathrm{L}}(\tau), \mathcal{B}_{\mathrm{R}}-\mathcal{B}_{\mathrm{L}}\right] / 2}-\kappa^{2}=\left\langle e^{\sum_{q} \frac{\lambda_{q}^{*}}{\omega_{q}}\left(1+e^{+\mathrm{i} \omega_{q} \tau}\right) a_{q}^{\dagger}-\frac{\lambda_{q}}{\omega_{q}}\left(1+e^{-\mathrm{i} \omega_{q} \tau}\right) a_{q}}\right\rangle e^{+2 \mathrm{i} \Phi} \\
& \times e^{\mathrm{i} \sum_{q} \frac{\left|\lambda_{q}\right|^{2}}{\omega_{q}^{2}} \sin \left(\omega_{q} \tau\right)}-\kappa^{2}=e^{+2 \mathrm{i} \Phi} e^{-\sum_{q} \frac{\left|\lambda_{q}\right|^{2}}{\omega_{q}^{2}}\left[\left(1+n_{\mathrm{B}}\left(\omega_{q}\right)\right) e^{-\mathrm{i} \omega_{q} \tau}+n_{\mathrm{B}}\left(\omega_{q}\right) e^{+\mathrm{i} \omega_{q} \tau}\right]} e^{-\sum_{q} \frac{\left|\lambda_{q}\right|^{2}}{\omega_{q}^{2}}\left(1+2 n_{\mathrm{B}}\left(\omega_{q}\right)\right)}-\kappa^{2} \\
= & \kappa^{2}\left[e^{-\sum_{q} \frac{\left|\lambda_{q}\right|^{2}}{\omega_{q}^{2}}\left[\left(1+n_{\mathrm{B}}\left(\omega_{q}\right)\right) e^{-\mathrm{i} \omega_{q} \tau}+n_{\mathrm{B}}\left(\omega_{q}\right) e^{+\mathrm{i} \omega_{q} \tau}\right]}-1\right],
\end{aligned}
$$

where $\lambda_{q}=h_{q L}-h_{q R}$. The bath correction function $\mathcal{C}_{66}(\tau)$ can be obtained via $\mathcal{C}_{66}(\tau) \hat{=} \mathcal{C}_{55}^{*}(-\tau)$. We show explicitly that $\mathcal{C}_{56}(\tau)$ is given by Eq. (49),

$$
\begin{aligned}
\mathcal{C}_{56}(\tau)= & \left\langle e^{\mathcal{B}_{\mathrm{R}}(\tau)-\mathcal{B}_{\mathrm{L}}(\tau)-\left(\mathcal{B}_{\mathrm{R}}-\mathcal{B}_{\mathrm{L}}\right)}\right\rangle \\
& \times e^{-\left[\mathcal{B}_{\mathrm{R}}(\tau)-\mathcal{B}_{\mathrm{L}}(\tau), \mathcal{B}_{\mathrm{R}}-\mathcal{B}_{\mathrm{L}}\right] / 2}-|\kappa|^{2} \\
= & \left\langle e^{\sum_{q} \frac{\lambda_{q}^{*}}{\omega_{q}}\left(e^{+\mathrm{i} \omega_{q} \tau}-1\right) a_{q}^{\dagger}-\frac{\lambda_{q}}{\omega_{q}}\left(e^{-\mathrm{i} \omega_{q} \tau}-1\right) a_{q}}\right\rangle \\
& \times e^{-\mathrm{i} \sum_{q} \frac{\left|\lambda_{q}\right|^{2}}{\omega_{q}^{2}} \sin \left(\omega_{q} \tau\right)}-|\kappa|^{2} \\
= & |\kappa|^{2}\left[e^{+\sum_{q} \frac{\left|\lambda_{q}\right|^{2}}{\omega_{q}^{2}}\left[\left(1+n_{\mathrm{B}}\left(\omega_{q}\right)\right) e^{-\mathrm{i} \omega_{q} \tau}+n_{\mathrm{B}}\left(\omega_{q}\right) e^{+\mathrm{i} \omega_{q} \tau}\right]}-1\right]
\end{aligned}
$$

The bath correction function $\mathcal{C}_{65}(\tau)$ can be obtained via the KMS-condition, yielding $\mathcal{C}_{56}(\tau) \hat{=} \mathcal{C}_{65}(\tau)$.

\section{APPENDIX E: SYMMETRIES IN THE CHARACTERISTIC POLYNOMIALS}

To show these symmetries, we show separate symmetries of the terms in the characteristic polynomial.

First, we note that trivially, the combination $\mathcal{L}_{23} \mathcal{L}_{32}$ does not depend on counting fields and is thus, by construction, inert to symmetry transformations of type (63).

Second, one can directly show that terms of the form $\mathcal{L}_{12} \mathcal{L}_{21}, \mathcal{L}_{13} \mathcal{L}_{31}, \mathcal{L}_{24} \mathcal{L}_{42}$, and $\mathcal{L}_{34} \mathcal{L}_{43}$ are also invariant under such transformations. We only show this explicitly for the first combination (the proof is analogous for the other terms), where we have

$$
\begin{aligned}
& \mathcal{L}_{12}=\sum_{\boldsymbol{n}}\left(\Gamma_{\mathrm{L}}^{0-,-\boldsymbol{n}} e^{-\mathrm{i} \chi} e^{-\mathrm{i} \xi\left(\varepsilon_{-}-\varepsilon_{0}+\boldsymbol{n} \cdot \boldsymbol{\Omega}\right)} e^{+\mathrm{i} \phi \boldsymbol{n} \cdot \boldsymbol{\Omega}}+\Gamma_{\mathrm{R}}^{0-,-\boldsymbol{n}} e^{+\mathrm{i} \phi \boldsymbol{n} \cdot \boldsymbol{\Omega}}\right), \\
& \mathcal{L}_{21}=\sum_{\boldsymbol{n}}\left(\Gamma_{\mathrm{L}}^{-0,+\boldsymbol{n}} e^{+\mathrm{i} \chi} e^{+\mathrm{i} \xi\left(\varepsilon_{-}-\varepsilon_{0}+\boldsymbol{n} \cdot \boldsymbol{\Omega}\right)} e^{-\mathrm{i} \phi \boldsymbol{n} \cdot \boldsymbol{\Omega}}+\Gamma_{\mathrm{R}}^{-0,+\boldsymbol{n}} e^{-\mathrm{i} \phi \boldsymbol{n} \cdot \boldsymbol{\Omega}}\right) .
\end{aligned}
$$

We can use the detailed balance relations (62) to rewrite, e.g., the first matrix element as (now keeping the counting fields explicitly),

$$
\begin{aligned}
\mathcal{L}_{12}(\chi, \xi, \phi) & =\sum_{\boldsymbol{n}}\left(\Gamma_{\mathrm{L}}^{-0,+\boldsymbol{n}} e^{-\mathrm{i} \chi} e^{-\mathrm{i} \xi\left(\varepsilon_{-}-\varepsilon_{0}+\boldsymbol{n} \cdot \boldsymbol{\Omega}\right)} e^{+\mathrm{i} \phi \boldsymbol{n} \cdot \boldsymbol{\Omega}} e^{+\beta_{\mathrm{L}}\left(\varepsilon_{-}-\varepsilon_{0}-\mu_{\mathrm{L}}+\boldsymbol{n} \cdot \boldsymbol{\Omega}\right)} e^{-\beta_{\mathrm{ph}} \boldsymbol{n} \cdot \boldsymbol{\Omega}}+\Gamma_{\mathrm{R}}^{-0,+\boldsymbol{n}} e^{+\beta_{\mathrm{R}}\left(\varepsilon_{-}-\varepsilon_{0}-\mu_{\mathrm{R}}+\boldsymbol{n} \cdot \boldsymbol{\Omega}\right)} e^{-\beta_{\mathrm{ph}} \boldsymbol{n} \cdot \boldsymbol{\Omega}}\right) \\
& =e^{\beta_{\mathrm{R}}\left(\varepsilon_{-}-\varepsilon_{0}-\mu_{R}\right)} \mathcal{L}_{21}\left(-\chi+\mathrm{i}\left(\beta_{\mathrm{L}} \mu_{\mathrm{L}}-\beta_{\mathrm{R}} \mu_{\mathrm{R}}\right),-\xi+\mathrm{i}\left(\beta_{\mathrm{R}}-\beta_{\mathrm{L}}\right),-\phi+\mathrm{i}\left(\beta_{\mathrm{R}}-\beta_{\mathrm{ph}}\right)\right)
\end{aligned}
$$

With the short-hand notation $\mathcal{L}_{i j}^{-}=\mathcal{L}_{i j}(-\chi)$ and $\overline{\mathcal{L}}_{i j}$ $=\mathcal{L}_{i j}(\chi+\mathrm{i} \Delta)$ where $\boldsymbol{\Delta}=\left(\beta_{\mathrm{L}} \mu_{\mathrm{L}}-\beta_{\mathrm{R}} \mu_{\mathrm{R}}, \beta_{\mathrm{R}}-\beta_{\mathrm{L}}, \beta_{\mathrm{R}}-\beta_{\mathrm{ph}}\right)$, we can summarize the relations

$$
\begin{aligned}
& \mathcal{L}_{12}^{-}=e^{+\beta_{\mathrm{R}}\left(\varepsilon_{-}-\varepsilon_{0}-\mu_{\mathrm{R}}\right)} \overline{\mathcal{L}}_{21}, \\
& \mathcal{L}_{21}^{-}=e^{-\beta_{\mathrm{R}}\left(\varepsilon_{-}-\varepsilon_{0}-\mu_{\mathrm{R}}\right)} \overline{\mathcal{L}}_{12}, \\
& \mathcal{L}_{13}^{-}=e^{+\beta_{\mathrm{R}}\left(\varepsilon_{+}-\varepsilon_{0}-\mu_{\mathrm{R}}\right)} \overline{\mathcal{L}}_{31}, \\
& \mathcal{L}_{31}^{-}=e^{-\beta_{\mathrm{R}}\left(\varepsilon_{+}-\varepsilon_{0}-\mu_{\mathrm{R}}\right)} \overline{\mathcal{L}}_{13}, \\
& \mathcal{L}_{24}^{-}=e^{+\beta_{\mathrm{R}}\left(\varepsilon_{2}-\varepsilon_{-}-\mu_{\mathrm{R}}\right)} \overline{\mathcal{L}}_{42}, \\
& \mathcal{L}_{42}^{-}=e^{-\beta_{\mathrm{R}}\left(\varepsilon_{2}-\varepsilon_{-}-\mu_{\mathrm{R}}\right)} \overline{\mathcal{L}}_{24}, \\
& \mathcal{L}_{34}^{-}=e^{+\beta_{\mathrm{R}}\left(\varepsilon_{2}-\varepsilon_{+}-\mu_{\mathrm{R}}\right)} \overline{\mathcal{L}}_{43}, \\
& \mathcal{L}_{43}^{-}=e^{-\beta_{\mathrm{R}}\left(\varepsilon_{2}-\varepsilon_{+}-\mu_{\mathrm{R}}\right)} \overline{\mathcal{L}}_{34},
\end{aligned}
$$

such that, e.g., products of the form $\mathcal{L}_{12} \mathcal{L}_{21}$ are invariant under the transformations (63), i.e., $\mathcal{L}_{12}^{-} \mathcal{L}_{21}^{-}=\overline{\mathcal{L}}_{12} \overline{\mathcal{L}}_{21}$.

Third, we consider combinations of three off-diagonal matrix elements by noting the additional symmetry

$$
\begin{aligned}
& \mathcal{L}_{23}^{-}=e^{+\beta_{\mathrm{R}}\left(\varepsilon_{+}-\varepsilon_{-}\right)} \overline{\mathcal{L}}_{32}, \\
& \mathcal{L}_{32}^{-}=e^{-\beta_{\mathrm{R}}\left(\varepsilon_{+}-\varepsilon_{-}\right)} \overline{\mathcal{L}}_{23},
\end{aligned}
$$

which together with the symmetries in Eq. (E4) can be used to show that in the characteristic polynomial (61) the terms with three off-diagonal matrix elements are also inert under the transformations (63), i.e.,

$$
\begin{aligned}
& \mathcal{L}_{23}^{-} \mathcal{L}_{34}^{-} \mathcal{L}_{42}^{-}+\mathcal{L}_{24}^{-} \mathcal{L}_{43}^{-} \mathcal{L}_{32}^{-}=\overline{\mathcal{L}}_{23} \overline{\mathcal{L}}_{34} \overline{\mathcal{L}}_{42}+\overline{\mathcal{L}}_{24} \overline{\mathcal{L}}_{43} \overline{\mathcal{L}}_{32}, \\
& \mathcal{L}_{12}^{-} \mathcal{L}_{23}^{-} \mathcal{L}_{31}^{-}+\mathcal{L}_{13}^{-} \mathcal{L}_{32}^{-} \mathcal{L}_{21}^{-}=\overline{\mathcal{L}}_{12} \overline{\mathcal{L}}_{23} \overline{\mathcal{L}}_{31}+\overline{\mathcal{L}}_{13} \overline{\mathcal{L}}_{32} \overline{\mathcal{L}}_{21}
\end{aligned}
$$


Finally, we note that the terms $\mathcal{L}_{12} \mathcal{L}_{21} \mathcal{L}_{34} \mathcal{L}_{43}$ and $\mathcal{L}_{13} \mathcal{L}_{31} \mathcal{L}_{24} \mathcal{L}_{42}$ can be treated similarly to the terms with just two off-diagonal matrix elements, and that the last two terms in the characteristic polynomial (61) obey

$$
\begin{aligned}
& \mathcal{L}_{12}^{-} \mathcal{L}_{24}^{-} \mathcal{L}_{43}^{-} \mathcal{L}_{31}^{-}+\mathcal{L}_{13}^{-} \mathcal{L}_{34}^{-} \mathcal{L}_{42}^{-} \mathcal{L}_{21}^{-} \\
& \quad=\overline{\mathcal{L}}_{12} \overline{\mathcal{L}}_{24} \overline{\mathcal{L}}_{43} \overline{\mathcal{L}}_{31}+\overline{\mathcal{L}}_{13} \overline{\mathcal{L}}_{34} \overline{\mathcal{L}}_{42} \overline{\mathcal{L}}_{21}
\end{aligned}
$$

which can be shown with Eqs. (E3).

${ }^{1}$ J. Gorman, D. G. Hasko, and D. A. Williams, "Charge-qubit operation of an isolated double quantum dot,” Phys. Rev. Lett. 95, 090502 (2005).

${ }^{2}$ B. Trauzettel, D. V. Bulaev, D. Loss, and G. Burkard, "Spin qubits in graphene quantum dots," Nat. Phys. 3, 192 (2007).

${ }^{3}$ J. Claudon, J. Bleuse, N. S. Malik, M. Bazin, P. Jaffrennou, N. Gregersen, C. Sauvan, P. Lalanne, and J.-M. Gerard, "A highly efficient single-photon source based on a quantum dot in a photonic nanowire," Nat. Photonics $\mathbf{4}$, 174 (2010).

${ }^{4}$ M. J. Holmes, K. Choi, S. Kako, M. Arita, and Y. Arakawa, "Roomtemperature triggered single photon emission from a III-nitride sitecontrolled nanowire quantum dot," Nano Lett. 14(2), 982-986 (2014).

${ }^{5}$ Y.-Y. Liu, K. D. Petersson, J. Stehlik, J. M. Taylor, and J. R. Petta, "Photon emission from a cavity-coupled double quantum dot," Phys. Rev. Lett. 113, 036801 (2014).

${ }^{6}$ S. Ballmann, R. Härtle, P. B. Coto, M. Elbing, M. Mayor, M. R. Bryce, M. Thoss, and H. B. Weber, "Experimental evidence for quantum interference and vibrationally induced decoherence in single-molecule junctions," Phys. Rev. Lett. 109, 056801 (2012).

${ }^{7}$ R. Härtle, M. Butzin, and M. Thoss, "Vibrationally induced decoherence in single-molecule junctions," Phys. Rev. B 87, 085422 (2013).

${ }^{8}$ J. K. Gamble, M. Friesen, S. N. Coppersmith, and X. Hu, "Two-electron dephasing in single Si and gaas quantum dots," Phys. Rev. B 86, 035302 (2012).

${ }^{9}$ P. Kaer and J. Mørk, "Decoherence in semiconductor cavity QED systems due to phonon couplings," Phys. Rev. B 90, 035312 (2014).

${ }^{10} \mathrm{~T}$. Brandes and B. Kramer, "Spontaneous emission of phonons by coupled quantum dots," Phys. Rev. Lett. 83, 3021-3024 (1999).

${ }^{11}$ A. Ueda, O. Entin-Wohlman, M. Eto, and A. Aharony, "Phonon spectroscopy by electric measurements of coupled quantum dots," Phys. Rev. B 82, 245317 (2010).

${ }^{12}$ P. Roulleau, S. Baer, T. Choi, F. Molitor, J. Güttinger, T. Müller, S. Dröscher, K. Ensslin, and T. Ihn, "Coherent electronphonon coupling in tailored quantum systems," Nat. Commun. 2, 239 (2011).

${ }^{13}$ K. J. Franke and J. I. Pascual, "Effects of electron-vibration coupling in transport through single molecules," J. Phys.: Condens. Matter 24(39), 394002 (2012).

${ }^{14} \mathrm{~J}$. Koch and F. von Oppen, "Franck-Condon blockade and giant Fano factors in transport through single molecules," Phys. Rev. Lett. 94, 206804 (2005).

${ }^{15}$ J. Koch, F. von Oppen, and A. V. Andreev, "Theory of the Franck-Condon blockade regime," Phys. Rev. B 74, 205438 (2006).

${ }^{16}$ R. Leturcq, C. Stampfer, K. Inderbitzin, L. Durrer, C. Hierold, E. Mariani, M. G. Schultz, F. von Oppen, and K. Ensslin, "Franck-Condon blockade in suspended carbon nanotube quantum dots," Nat. Phys. 5(5), 327-331P (2009).

${ }^{17}$ A. Donabidowicz-Kolkowska and C. Timm, "Spectroscopy of the transitionrate matrix for molecular junctions: Dynamics in the Franck-Condon regime," New J. Phys. 14(10), 103050 (2012).

${ }^{18}$ R. Hützen, S. Weiss, M. Thorwart, and R. Egger, "Iterative summation of path integrals for nonequilibrium molecular quantum transport," Phys. Rev. B 85, 121408 (2012).

${ }^{19}$ D. H. Santamore, N. Lambert, and F. Nori, "Vibrationally mediated transport in molecular transistors," Phys. Rev. B 87, 075422 (2013).

${ }^{20} \mathrm{C}$. Timm and M. Di Ventra, "Molecular neuron based on the Franck-Condon blockade," Nanotechnology 24(38), 384001 (2013).

${ }^{21}$ J. C. Cuevas and E. Scheer, Molecular Electronics - An Introduction to Theory and Experiment (World Scientific, 2010).

${ }^{22}$ R. Volkovich, R. Hartle, M. Thoss, and U. Peskin, "Bias-controlled selective excitation of vibrational modes in molecular junctions: A route towards mode-selective chemistry," Phys. Chem. Chem. Phys. 13, 14333-14349 (2011).

${ }^{23} \mathrm{D}$. Segal, "Thermoelectric effect in molecular junctions: A tool for revealing transport mechanisms," Phys. Rev. B 72, 165426 (2005).
${ }^{24}$ M. Galperin, A. Nitzan, and M. A. Ratner, "Inelastic effects in molecular junction transport: Scattering and self-consistent calculations for the seebeck coefficient," Mol. Phys. 106(2-4), 397-404 (2008).

${ }^{25}$ Y. Dubi and M. Di Ventra, "Thermoelectric effects in nanoscale junctions," Nano Lett. 9(1), 97-101 (2009).

${ }^{26}$ Y. Dubi and M. Di Ventra, "Colloquium: Heat flow and thermoelectricity in atomic and molecular junctions," Rev. Mod. Phys. 83, 131-155 (2011).

${ }^{27}$ R. Härtle and M. Thoss, "Vibrational instabilities in resonant electron transport through single-molecule junctions," Phys. Rev. B 83, 125419 (2011).

${ }^{28}$ B. Rutten, M. Esposito, and B. Cleuren, "Reaching optimal efficiencies using nanosized photoelectric devices," Phys. Rev. B 80, 235122 (2009).

${ }^{29}$ O. Entin-Wohlman, Y. Imry, and A. Aharony, "Three-terminal thermoelectric transport through a molecular junction," Phys. Rev. B 82, 115314 (2010).

${ }^{30}$ R. Sánchez, B. Sothmann, A. N. Jordan, and M. Büttiker, "Correlations of heat and charge currents in quantum-dot thermoelectric engines," New J. Phys. 15(12), 125001 (2013).

${ }^{31}$ B. Sothmann, R. Sánchez, and A. N. Jordan, "Thermoelectric energy harvesting with quantum dots," Nanotechnology 26(3), 032001 (2015).

${ }^{32}$ M. Esposito, K. Lindenberg, and C. Van den Broeck, "Thermoelectric efficiency at maximum power in a quantum dot," EPL 85(6), 60010 (2009).

${ }^{33}$ M. Galperin, K. Saito, A. V. Balatsky, and A. Nitzan, "Cooling mechanisms in molecular conduction junctions," Phys. Rev. B 80(11), 115427 (2009).

${ }^{34}$ L. Arrachea, N. Bode, and F. von Oppen, "Vibrational cooling and thermoelectric response of nanoelectromechanical systems," Phys. Rev. B 90, 125450 (2014).

${ }^{35} \mathrm{~F}$. Haupt, T. Novotný, and W. Belzig, "Phonon-assisted current noise in molecular junctions," Phys. Rev. Lett. 103, 136601 (2009).

${ }^{36}$ T.-H. Park and M. Galperin, "Self-consistent full counting statistics of inelastic transport," Phys. Rev. B 84, 205450 (2011).

${ }^{37}$ M. Galperin, A. Nitzan, and M. A. Ratner, "Resonant inelastic tunneling in molecular junctions," Phys. Rev. B 73, 045314 (2006).

${ }^{38}$ L. Nicolin and D. Segal, "Quantum fluctuation theorem for heat exchange in the strong coupling regime," Phys. Rev. B 84, 161414 (2011).

${ }^{39}$ L. Nicolin and D. Segal, "Non-equilibrium spin-boson model: Counting statistics and the heat exchange fluctuation theorem," J. Chem. Phys. 135(16), 164106 (2011).

${ }^{40}$ J. Ren, J.-X. Zhu, J. E. Gubernatis, C. Wang, and B. Li, "Thermoelectric transport with electron-phonon coupling and electron-electron interaction in molecular junctions," Phys. Rev. B 85, 155443 (2012).

${ }^{41}$ T. Koch, J. Loos, and H. Fehske, "Thermoelectric effects in molecular quantum dots with contacts," Phys. Rev. B 89, 155133 (2014).

${ }^{42}$ A. Benyamini, A. Hamo, S. Viola Kusminskiy, F. von Oppen, and S. Ilani, "Real-space tailoring of the electron-phonon coupling in ultraclean nanotube mechanical resonators," Nat. Phys. 10, 081412 (2014).

${ }^{43}$ T. Holstein, "Studies of polaron motion: Part I. The molecular-crystal model," Ann. Phys. 8(3), 325-342 (1959).

${ }^{44}$ P. W. Anderson, "Localized magnetic states in metals," Phys. Rev. 124, 41-53 (1961)

${ }^{45}$ R. C. Monreal and A. Martin-Rodero, "Equation of motion approach to the Anderson-Holstein Hamiltonian,” Phys. Rev. B 79, 115140 (2009).

${ }^{46}$ M. Galperin, M. A. Ratner, and A. Nitzan, "Molecular transport junctions: Vibrational effects," J. Phys.: Condens. Matter 19, 103201 (2007).

${ }^{47}$ C. Schinabeck, R. Härtle, H. B. Weber, and M. Thoss, "Current noise in single-molecule junctions induced by electronic-vibrational coupling," Phys. Rev. B 90, 075409 (2014).

${ }^{48}$ D. Kast, L. Kecke, and J. Ankerhold, "Charge transfer through single molecule contacts: How reliable are rate descriptions?,” Beilstein J. Nanotechnol. 2, 416 (2011).

${ }^{49}$ R. Avriller and A. Levy Yeyati, "Electron-phonon interaction and full counting statistics in molecular junctions," Phys. Rev. B 80, 041309 (2009).

${ }^{50}$ D. F. Urban, R. Avriller, and A. Levy Yeyati, "Nonlinear effects of phonon fluctuations on transport through nanoscale junctions," Phys. Rev. B 82, 121414 (2010).

${ }^{51}$ C. Wang, J. Ren, B. W. Li, and Q. H. Chen, "Quantum transport of double quantum dots coupled to an oscillator in arbitrary strong coupling regime," Eur. Phys. J. B 85(3), 110 (2012).

${ }^{52}$ A. A. Dzhioev and D. S. Kosov, "Nonequilibrium perturbation theory in Liouville-Fock space for inelastic electron transport," J. Phys.: Condens. Matter 24, 225304 (2012).

${ }^{53}$ R. Härtle, C. Benesch, and M. Thoss, "Vibrational nonequilibrium effects in the conductance of single molecules with multiple electronic states," Phys. Rev. Lett. 102, 146801 (2009).

${ }^{54}$ M. Leijnse and M. R. Wegewijs, "Kinetic equations for transport through single-molecule transistors," Phys. Rev. B 78, 235424 (2008). 
${ }^{55} \mathrm{H}$. Wang and M. Thoss, "Numerically exact quantum dynamics for indistinguishable particles: The multilayer multiconfiguration time-dependent Hartree theory in second quantization representation,' J. Chem. Phys. 131, 024114 (2009).

${ }^{56}$ K. F. Albrecht, H. Wang, L. Mühlbacher, M. Thoss, and A. Komnik, "Bistability signatures in nonequilibrium charge transport through molecular quantum dots," Phys. Rev. B 86, 081412 (2012).

${ }^{57}$ E. Y. Wilner, H. Wang, M. Thoss, and E. Rabani, "Nonequilibrium quantum systems with electron-phonon interactions: Transient dynamics and approach to steady state," Phys. Rev. B 89, 205129 (2014)

${ }^{58}$ L. Mühlbacher and E. Rabani, "Real-time path integral approach to nonequilibrium many-body quantum systems," Phys. Rev. Lett. 100, 176403 (2008).

${ }^{59}$ M. Schiró and M. Fabrizio, "Real-time diagrammatic Monte Carlo for nonequilibrium quantum transport,” Phys. Rev. B 79, 153302 (2009).

${ }^{60}$ L. Simine and D. Segal, "Path-integral simulations with fermionic and bosonic reservoirs: Transport and dissipation in molecular electronic junctions," J. Chem. Phys. 138, 214111 (2013).

${ }^{61}$ S. Weiler, A. Ulhaq, S. M. Ulrich, D. Richter, M. Jetter, P. Michler, C. Roy, and S. Hughes, "Phonon-assisted incoherent excitation of a quantum dot and its emission properties,” Phys. Rev. B 86, 241304 (2012).

${ }^{62}$ S. Maier, T. L. Schmidt, and A. Komnik, "Charge transfer statistics of a molecular quantum dot with strong electron-phonon interaction," Phys. Rev. B 83, 085401 (2011)

${ }^{63}$ S. Walter, B. Trauzettel, and T. L. Schmidt, "Transport properties of double quantum dots with electron-phonon coupling," Phys. Rev. B 88, 195425 (2013).

${ }^{64}$ G. Schaller, T. Krause, T. Brandes, and M. Esposito, "Single-electron transistor strongly coupled to vibrations: Counting statistics and fluctuation theorem," New J. Phys. 15, 033032 (2013).

${ }^{65}$ G. E. Crooks, "Entropy production fluctuation theorem and the nonequilibrium work relation for free energy differences,” Phys. Rev. E 60, 2721-2726 (1999).

${ }^{66} \mathrm{U}$. Seifert, "Entropy production along a stochastic trajectory and an integral fluctuation theorem," Phys. Rev. Lett. 95, 040602 (2005).

${ }^{67}$ M. Esposito, U. Harbola, and S. Mukamel, "Fluctuation theorem for counting statistics in electron transport through quantum junctions," Phys. Rev. B 75, 155316 (2007)

${ }^{68}$ M. Esposito and C. Van den Broeck, "Three faces of the second law. I. Master equation formulation,” Phys. Rev. E 82(1), 011143 (2010).
${ }^{69}$ G. D. Mahan, Many-Particle Physics (Springer, Netherlands, 2000)

${ }^{70} \mathrm{~T}$. Brandes, "Coherent and collective quantum optical effects in mesoscopic systems," Phys. Rep. 408, 315-474 (2005).

${ }^{71}$ A. Jovchev and F. B. Anders, "Influence of vibrational modes on quantum transport through a nanodevice," Phys. Rev. B 87, 195112 (2013).

${ }^{72}$ R. Härtle and M. Thoss, "Resonant electron transport in single-molecule junctions: Vibrational excitation, rectification, negative differential resistance, and local cooling," Phys. Rev. B 83, 115414 (2011).

${ }^{73}$ A. S. Alexandrov, A. M. Bratkovsky, and R. Stanley Williams, "Bistable tunneling current through a molecular quantum dot," Phys. Rev. B 67, 075301 (2003).

${ }^{74}$ J. Koch, E. Sela, Y. Oreg, and F. von Oppen, "Nonequilibrium chargeKondo transport through negative- $U$ molecules," Phys. Rev. B 75, 195402 (2007).

${ }^{75}$ H.-P. Breuer and F. Petruccione, The Theory of Open Quantum Systems (Oxford University Press, Oxford, 2002).

${ }^{76}$ G. Schaller, Open Quantum Systems Far from Equilibrium (Springer, 2014).

${ }^{77}$ G. Schaller, G. Kießlich, and T. Brandes, "Transport statistics of interacting double dot systems: Coherent and non-Markovian effects,' Phys. Rev. B 80, 245107 (2009).

${ }^{78}$ P. Zedler, G. Schaller, G. Kießlich, C. Emary, and T. Brandes, "Weak coupling approximations in non-Markovian transport,” Phys. Rev. B 80, 045309 (2009).

${ }^{79}$ T. Brandes and N. Lambert, "Steering of a bosonic mode with a double quantum dot," Phys. Rev. B 67, 125323 (2003).

${ }^{80}$ T. Brandes, R. Aguado, and G. Platero, "Charge transport through open driven two-level systems with dissipation,” Phys. Rev. B 69, 205326 (2004).

${ }^{81}$ C. Wang, J. Ren, B. W. Li, and Q. H. Chen, "Quantum transport of double quantum dots coupled to an oscillator in arbitrary strong coupling regime," Eur. Phys. J. B 85, 110 (2012)

${ }^{82}$ D. H. Santamore, N. Lambert, and F. Nori, "Vibrationally mediated transport in molecular transistors," Phys. Rev. B 87, 075422 (2013).

${ }^{83}$ J. Koch and F. von Oppen, "Franck-Condon blockade and giant Fano factors in transport through single molecules," Phys. Rev. Lett. 94, 206804 (2005).

${ }^{84}$ T. Krause, G. Schaller, and T. Brandes, "Incomplete current fluctuation theorems for a four-terminal model," Phys. Rev. B 84, 195113 (2011).

${ }^{85}$ A. Gomez-Marin and J. M. Sancho, "Tight coupling in thermal brownian motors," Phys. Rev. E 74, 062102 (2006). 